\title{
Harsanyi support levels solutions
}

\author{
Manfred Besner ${ }^{1}$
}

Accepted: 29 May 2021 / Published online: 7 August 2021

(C) The Author(s) 2021

\begin{abstract}
We introduce a new class of values for TU-games (games with transferable utility) with a level structure, called LS-games. A level structure is a hierarchical structure where each level corresponds to a partition of the player set, which becomes increasingly coarse from the trivial partition containing only singletons to the partition containing only the grand coalition. The new values, called Harsanyi support levels solutions, extend the Harsanyi solutions for LS-games. As an important subset of the class of these values, we present the class of weighted Shapley support levels values as a further result. The values from this class extend the weighted Shapley values for LS-games and contain the Shapley levels value as a special case. Axiomatizations of the studied classes are provided.
\end{abstract}

Keywords Cooperative game · Level structure · (Weighted) Shapley (levels) value $\cdot$ Harsanyi set $\cdot$ Dividends

\section{Introduction}

Many institutions, companies, governments, and so on are organized in hierarchical structures. Typically, there is one unit at the top. In the following levels, each unit of the parent level is divided into two or more subordinate units, which usually have a lower rank than the higher ones. We can see a similar organizational structure in supply chains in some respects, and queuing problems or electricity and other networks often have hierarchical structures as well. Effectiveness increases by sharing or pooling physical objects, resources, and information. A central characteristic of all these forms of organization is that a cooperating unit can itself be an actor to gain advantages of cooperation for the members of the unit. The question arises: how should we share the benefits and allocate the costs?

Manfred Besner

manfred.besner@hft-stuttgart.de

1 Department of Geomatics, Computer Science and Mathematics, HFT Stuttgart, University of Applied Sciences, Schellingstr. 24, 70174 Stuttgart, Germany 
To distribute the profits of cooperating coalitions, the application of a cooperative game seems to be a natural approach. Winter (1989) defined a model for cooperative games with a level structure (LS-games). A level structure comprises a sequence of coalition structures (the levels). At each level, the player set is partitioned into components where each higher level is coarser than the previous one (see also Fig. 1). Winter's value (Winter, 1989) for LS-games, we call it Shapley levels value, extends the Owen value (Owen, 1977), which is itself an extension of the Shapley value (Shapley, 1953b). This value satisfies adaptations of the symmetry axioms, satisfied by the Owen value, for values for LS-games.

To treat symmetric players differently when there are exogenous weights for the players, Shapley (1953a) introduced the weighted Shapley values. Vidal-Puga (2012) established a value for games with a coalition structure with weights given by the size of the coalitions. With a step by step top-down algorithm, Gómez-Rúa and Vidal-Puga (2011) extended it for games with a level structure. Besner (2019) generalized this value to the class of the weighted Shapley hierarchy levels values for arbitrary, exogenously given, weights. These values satisfy an extension of the consistency property of the weighted Shapley values in Hart and Mas-Colell (1989). Interestingly, the class of the weighted Shapley hierarchy levels values contains the Shapley levels value but the values from this class do not satisfy the null player axiom in general.

The weighted values for games with a coalition structure in Levy and Mclean (1989) and McLean (1991) behave the other way round; they satisfy the null player property but do not correspond to a consistency property in the above sense. Levy and Mclean (1989) examine several classes of weighted values for games with a coalition structure which use the same weight system as the weighted Shapley values: either for the players within a component or the components themselves if the components act as players. The combined use of such a weight system, both for players and components, is only mentioned. This latter class of extensions of the weighted Shapley values and an extension of the class of random order values (Weber, 1988) for games with a coalition structure is discussed in McLean (1991). Dragan (1992) called McLean's extensions of the weighted Shapley values McLean weighted coalition structure values. He presented a formula for them related to that of the Owen value. For a fixed coalition structure, these values coincide with a multiweighted Shapley value (Dragan, 1992).

Harsanyi (1959) introduced a new perspective on coalition functions. He used socalled (Harsanyi) dividends, assigned to all feasible coalitions of a player set according to the coalition function. Singletons receive the singleton worth as their dividend and the dividend of each larger coalition $S$ amounts to the worth of $S$ minus the sum of all dividends of the proper subcoalitions of $S$. The weighted Shapley values give the players as payoffs a share of the dividends from the coalitions in which they are members. Two players' shares of dividends from coalitions containing both players are always in the same ratio. In comparison, the Harsanyi solutions (Hammer et al., 1977; Vasil'ev, 1978) are more flexible. By these values, two players can receive dividend shares from coalitions, containing both, in various ratios for each coalition. 
In this article, we introduce the class of Harsanyi support levels solutions. To the best of our knowledge, the values of this class are the first values for games with level structure (LS-values) that extend the Harsanyi solutions. We represent each Harsanyi support levels solution by a formula with dividends. The coefficients in the formulas constitute a dividend sharing system, i. e., all coefficients are non-negative and amount to one for each coalition. According to the definition of a Harsanyi solution, each LS-value from this class corresponds to a Harsanyi solution for a fixed level structure. Therefore, Harsanyi solutions inherit all properties (adapted for LS-values) of these values for a fixed level structure.

Within the framework of LS-games, we can also consider the components of a level as players in so-called induced LS-games. Winter (1989) has shown that the Shapley levels value satisfies the level game property. This property means that the payoff to a component in an induced LS-game is equal to the sum of the payoffs to all players included in the component. We show that if an LS-value satisfies the level game property and coincides for a fixed level structure with a Harsanyi solution, the value is a member of the class of the Harsanyi support levels solutions. As a corollary, our main result extends an axiomatization of the class of the Harsanyi solutions by Vasil'ev (1981) and Derks et al. (2000) to a characterization of the class of the Harsanyi support levels solutions.

Since the Harsanyi solutions are no random order values in general, we cannot adopt the proof procedures for characterizations, e.g., in Winter (1989) or McLean (1991). We base our proofs on dividends where two new lemmas are a significant help.

As a further result, we present the class of the weighted Shapley support levels values as a proper subset of the class of the Harsanyi support levels solutions. The LS-values from this class coincide with the McLean weighted coalition structure values on a level structure with only three levels if we count the partition containing all singletons and the partition containing only the grand coalition as levels. To offer a characterization for this class, we adapt an axiomatization of the class of the weighted Shapley values in Nowak and Radzik (1995).

In the concluding section, it is briefly explained that the values presented in this article can have an interesting solidarity characteristic: players who have formed a group support the other group members even in situations where they are not active and do not expect any direct reward for their support, as with the weighted Shapley Hierarchy levels values. In this way, they differ fundamentally from solidaritybased solution concepts such as the equal division value, the equal surplus division value (Driessen and Funaki, 1991), the egalitarian Shapley values (Joosten, 1996) or the solidarity value (Nowak and Radzik, 1994) and the Shapley-solidarity value (Calvo and Gutiérrez, 2013).

The outline of the paper is structured as follows. Section 2 contains preliminaries. As the main part, we introduce in Sect. 3 the Harsanyi support levels solutions with an appropriate class axiomatization. In Sect. 4, we offer the weighted Shapley support levels values as a further result. A numerical example in Sect. 5 compares different values. Section 6 discusses the results and concludes with a fundamental principle of group solidarity which is satisfied by our new values. The Appendix 
(Sect. 7) provides all the proofs, related properties and lemmas, and shows the logical independence of the axioms in our axiomatizations.

\section{Preliminaries}

\subsection{TU-games}

Let $\mathbb{R}$ be the set of real numbers and $\mathbb{R}_{++}$the set of all positive real numbers. We fix a countably infinite set $\mathfrak{U}^{A}$, the universe of atomic players. The set $\mathfrak{U}$, the universe of all players ${ }^{1}$, is defined recursively:

(a) $i \in \mathfrak{U}$ for all $i \in \mathfrak{U}^{A}$.

(b) For all non-empty and finite subsets $N \subseteq \mathfrak{U}$, we have also $N \in \mathfrak{U}$.

(c) Nothing is in $\mathfrak{U}$ unless it can be shown to be there using (a) and (b).

We denote by $\mathcal{N}$ the set of all non-empty and finite subsets of $\mathfrak{U}$. A cooperative game with transferable utility (TU-game) is a pair $(N, v)$ such that $N \in \mathcal{N}$ is a set of players and $v: 2^{N} \rightarrow \mathbb{R}, v(\emptyset)=0$, a coalition function where $2^{N}$ is the power set of $N$. The subsets $S \subseteq N$ are called coalitions, $v(S)$ is the worth of coalition $S$, the set of all non-empty subsets of $S$ is denoted by $\Omega^{S}$, and the set of all TU-games with player set $N$ is denoted by $\mathbb{V}^{N}$.

Let $N \in \mathcal{N},(N, v) \in \mathbb{V}^{N}$ and $S \subseteq N$. The dividends $\Delta_{v}(S)$ (Harsanyi, 1959) are defined inductively by

$$
\Delta_{v}(S):=\left\{\begin{array}{c}
v(S)-\sum_{R \subsetneq S} \Delta_{v}(R), \text { if } S \in \Omega^{N}, \text { and } \\
0, \text { if } S=\emptyset .
\end{array}\right.
$$

A TU-game $\left(N, u_{T}\right) \in \mathbb{V}^{N}, T \in \Omega^{N}$, with $u_{T}(S):=1$ if $T \subseteq S$ and $u_{T}(S):=0$ otherwise for all $S \subseteq N$ is called a unanimity game. It is well known that any coalition function $v$ on $N$ has a unique representation

$$
v=\sum_{T \in \Omega^{N}} \Delta_{v}(T) u_{T} .
$$

$(N, v)$ is called totally positive (Vasil'ev, 1975) if $\Delta_{v}(T) \geq 0$ for all $T \subseteq N$. Player $i \in N$ is called a null player in $(N, v)$ if $v(S \cup\{i\})=v(S)$ for all $S \subseteq N \backslash\{i\}$; players $i, j \in N, i \neq j$, are called (mutually) dependent (Nowak and Radzik, 1995) in ( $N, v$ ) if $v(S \cup\{i\})=v(S)=v(S \cup\{j\})$ for all $S \subseteq N \backslash\{i, j\}$, which is equivalent to

$$
\Delta_{v}(S \cup\{k\})=0, k \in\{i, j\}, \text { for all } S \subseteq N \backslash\{i, j\} .
$$

For all $N \in \mathcal{N}$, a TU-value or solution $\phi$ is an operator that assigns to any $(N, v) \in$ $\mathbb{V}^{N}$ a payoff vector $\phi(N, v) \in \mathbb{R}^{N}$.

\footnotetext{
${ }^{1}$ Sets of players can also be players or their respective representatives (see, e.g., Kalai and Samet 1987 Section 7).
} 
By $W:=\left\{f: \mathfrak{U} \rightarrow \mathbb{R}_{++}\right\}, w_{i}:=w(i)$ for all $w \in W, i \in \mathfrak{U}$, we define the collection of all positive weight systems on $\mathfrak{U}$.

The following TU-values distribute the dividends of the coalitions proportionally to the weights of the players included in these coalitions. For all $N \in \mathcal{N},(N, v) \in$ $\mathbb{V}^{N}$, and each $w \in W$, the (positively) weighted Shapley value $S h^{w}$ (Shapley, $1953 \mathrm{a})$ assigns to any $(N, v)$ a vector in $\mathbb{R}^{N}$ defined as

$$
S h_{i}^{w}(N, v):=\sum_{S \subseteq N, S \ni i} \frac{w_{i}}{\sum_{j \in S} w_{j}} \Delta_{v}(S) \text { for all } i \in N .
$$

As a special case of a weighted Shapley value, all weights are equal, the Shapley value $S h$ (Shapley, 1953b) assigns to any $(N, v) \in \mathbb{V}^{N}$ a vector in $\mathbb{R}^{N}$ defined as

$$
S h_{i}(N, v):=\sum_{S \subseteq N, S \ni i} \frac{\Delta_{v}(S)}{|S|} \text { for all } i \in N .
$$

For all coalitions that include the same two players, for the weighted Shapley values, the dividend shares for these players are always in the same ratio. This need not be the case for the following TU-values, using sharing systems, where the ratio of the dividend shares of two players may also be different for two different coalitions containing both players. The collection $\Lambda$ of all sharing systems $\lambda \in \Lambda$ on $\mathcal{N}$ is defined by

$$
\Lambda:=\left\{\lambda=\left(\lambda_{S, i}\right)_{S \in \mathcal{N}, i \in S} \mid \sum_{i \in S} \lambda_{S, i}=1 \text { and } \lambda_{S, i} \geq 0 \text { for each } S \in \mathcal{N} \text { and all } i \in S\right\} .
$$

Hammer et al. (1977) and Vasil'ev (1978), independently of each other, defined a class of TU-values. While Hammer et al. (1977) and, e. g., Derks et al. (2000) this class call selectope ${ }^{2}$, Vasil'ev (1978), Vasil'ev and van der Laan (2002), and, e. g., del Pozo et al. (2011) refer to this class as Harsanyi set, abbreviated to $\mathcal{H}$. For all $N \in \mathcal{N},(N, v) \in \mathbb{V}^{N}$, and each sharing system $\lambda \in \Lambda$, the Harsanyi solution $H^{\lambda} \in$ $\mathcal{H}$ assigns to any $(N, v)$ a vector in $\mathbb{R}^{N}$ defined as

$$
H_{i}^{\lambda}(N, v):=\sum_{T \subseteq N, T \ni i} \lambda_{T, i} \Delta_{v}(T) \text { for all } i \in N .
$$

By (4), it is immediate that the class of the weighted Shapley values is a proper subset of the Harsanyi set.

\subsection{LS-games}

In the following, we have to restrict the amount of available player sets. We give some (a few times recursive) definitions where the underlying atomic players of players representing coalitions of players play an important role:

\footnotetext{
${ }^{2}$ Derks et al. (2000) provide in the first place a set-theoretic view of the selectope. Thereby, the selectope is the convex hull of so-called selector values and can be viewed as containing all the possible modes in which the Harsanyi dividends of a game can be distributed to the players.
} 
An atomic player $i \in \mathfrak{U}^{A}$ is called a sub-member of a player $j \in \mathfrak{U}$ if $i=j$ or $i$ is a sub-member of a player $k \in \mathfrak{U}, k \in j$. A player $i \in \mathfrak{U}$ is called a sub-playercoalition of a player $j \in \mathfrak{U}$ if $i=j$ or $i \in k, k$ is a sub-player-coalition of $j$. A player set $N \in \mathcal{N}$ is called sub-member-disjoint if no atomic player $i \in \mathfrak{U}^{A}$ is a submember of any two sub-player-coalitions $j, k, j \neq k$, such that $j, k \in \ell, \ell$ is a subplayer-coalition of $N .^{3}$ The set of all sub-member-disjoint player sets $N \in \mathcal{N}$ is denoted by $\mathcal{N}^{\text {dis }} \cdot 4$

For any $N \in \mathcal{N}^{\text {dis }}$, a coalition structure $\mathcal{B}$ on $N$ is a partition of the player set $N$, i.e., a collection of non-empty, pairwise disjoint, and mutually exhaustive subsets of $N$. Each $B \in \mathcal{B}$ is called a component and $\mathcal{B}(i)$ denotes the component that contains a player $i \in N$. A level structure (Winter, 1989) on $N$ is a finite sequence $\underline{\mathcal{B}}:=$ $\left\{\mathcal{B}^{0}, \ldots, \mathcal{B}^{h+1}\right\}$ of coalition structures $\mathcal{B}^{r}, 0 \leq r \leq h+1$, on $N$ such that (see also Fig. 1):

- $\mathcal{B}^{0}=\{\{i\}: i \in N\}$,

- $\mathcal{B}^{h+1}=\{N\}$, and

- for each $r, 0 \leq r \leq h, \mathcal{B}^{r}$ is a refinement of $\mathcal{B}^{r+1}$, i. e., $\mathcal{B}^{r}(i) \subseteq \mathcal{B}^{r+1}(i)$ for all $i \in N$.

$\mathcal{B}^{r}$ is called the $r$ th level of $\underline{\mathcal{B}}, \underline{\overline{\mathcal{B}}}:=\left\{B \mid B \in \mathcal{B}^{r}\right.$ for all $\left.\mathcal{B}^{r} \in \underline{\mathcal{B}}, 0 \leq r \leq h\right\}$ is the set of all components $B \in \mathcal{B}^{r}$ of all levels $\mathcal{B}^{r} \in \underline{\mathcal{B}}, 0 \leq r \leq h$, and $\mathbb{\mathbb { L }}^{N}$ denotes the set of all level structures on the player set $N$.

For each $B \in \mathcal{B}^{\ell}, 0 \leq \ell \leq r \leq h+1$, we denote by $\mathcal{B}^{r}(B)$ the component of the $r$ th level containing $B$ as a (not necessary proper) subset; $\mathcal{B}^{r}(B)$ is called an ancestor of $B$; if $r=\ell+1, \mathcal{B}^{r}(B)$ is also called parent of $B$. We call all components with the same parent $B \in \mathcal{B}^{r}, 1 \leq r \leq h+1$, children of $B$ and two different children of $B$ are called siblings in $\mathcal{B}^{r-1}$. We explicitly point out that a component, for different levels, can be its own child or parent at the same time.

If we regard the components of the $r$ th level, $0 \leq r \leq h$, as players and cancel all levels below the $r$ th level, we get the induced $r$ th level structure $\underline{\mathcal{B}^{r}}:=$ $\left\{\mathcal{B}^{r^{0}}, \ldots, \mathcal{B}^{r^{h+1-r}}\right\} \in \mathbb{E}^{\mathcal{B}^{r}}$ from $\underline{\mathcal{B}}=\left\{\mathcal{B}^{0}, \ldots, \mathcal{B}^{h+1}\right\}$. We give an illustrating example.

Example 2.1 Let $N=\{1,2,3\}$ and $\underline{\mathcal{B}}=\left\{\mathcal{B}^{0}, \mathcal{B}^{1}, \mathcal{B}^{2}\right\}$ be given by $\mathcal{B}^{0}=$ $\{\{1\},\{2\},\{3\}\}, \mathcal{B}^{1}=\{\{1,2\},\{3\}\}$, and $\mathcal{B}^{2}=\{N\}$. We regard, e.g., the components of the first level as players. Then, the induced first level structure $\underline{\mathcal{B}^{1}}=$

\footnotetext{
${ }^{3}$ For instance, $j:=\{\{i, k\},\{i, \ell\}\}, i, k, \ell \in \mathfrak{U}$, can not be a player of a sub-member-disjoint player set.

4 To exclude from the outset inconsistencies in the worths or in the weighting of coalitions and associated coalitions with the same sub-members, we will only consider player sets $N \in \mathcal{N}^{\text {dis }}$ for games with a level structure. For example, let $N:=\{i, j,\{i, j\}\}$ be a player set with three players where the third player is a coalition. In a TU-game $(N, v) \in \mathbb{V}^{N}$, it does not matter if the worth $v(\{i, j\})$ of the coalition $\{i, j\}$ of the two players $i, j \in N$ differs from the worth $v(\{\{i, j\}\})$ of the singleton $\{\{i, j\}\}$ of the player $\{i, j\} \in N$. For games with a level structure, we have an inconsistency in the induced first LS-game (see the definition below) if the coalition $\{i, j\}$ is there a player and we require that the induced first LS-game is given by (5).
} 
$\left\{\mathcal{B}^{1^{0}}, \mathcal{B}^{1^{1}}\right\} \quad$ from $\quad \underline{\mathcal{B}} \quad$ is $\quad$ given $\quad$ by $\quad \mathcal{B}^{1^{0}}=\{\{\{1,2\}\},\{\{3\}\}\}, \quad$ and $\mathcal{B}^{1}=\{\{\{1,2\},\{3\}\}\}$.

For each $N \in \mathcal{N}^{\text {dis }}$, a TU-game $(N, v) \in \mathbb{V}^{N}$ together with a level structure $\underline{\mathcal{B}} \in$ $\mathbb{Q}^{N}$ is an LS-game which is denoted by $(N, v, B)$. We denote the set of all LS-games on $N$ by $\mathbb{V} \mathbb{L}^{N}$.

If we want to stress the property that a coalition $T \in \Omega^{N}, T=\bigcup_{B \subset T, B \in \mathcal{B}^{r}} B$, is the union of components of the $r$ th level from $\underline{\mathcal{B}}$, we denote $T$ by $T^{r}$. Each such $T^{r}$ is an associated coalition to a coalition of all players $B \in \mathcal{B}^{r}, B \subseteq T^{r}$, in the induced $r$ th level structure, denoted by $\mathcal{T}^{r}:=\left\{B \in \mathcal{B}^{r}: B \subseteq T^{r}\right\}$ and vice versa. This means that a coalition and its associated coalition have the same sub-members. Thus, for each $N \in \mathcal{N}^{\text {dis }}$, each LS-game $(N, v, \underline{\mathcal{B}}) \in \mathbb{V} \mathbb{L}^{N}$, and each induced $r$ th level structure $\underline{\mathcal{B}^{r}}, 0 \leq r \leq h$, we have an associated LS-game $\left(\mathcal{B}^{r}, v^{r}, \underline{\mathcal{B}^{r}}\right) \in \mathbb{V} \mathbb{L}^{\mathcal{B}^{r}}$, called induced $\boldsymbol{r t h} \mathbf{L S}$-game ${ }^{5}$, with a coalition function $v^{r}$ which is given by

$$
v^{r}\left(\mathcal{T}^{r}\right):=v\left(T^{r}\right) \text { for all } \mathcal{T}^{r} \in \Omega^{\mathcal{B}^{r}} .
$$

This means that a coalition in an induced LS-game that has the same sub-members as a coalition from the original game also has the same worth as this associated coalition. E.g., for the TU-game $(N, v) \in \mathbb{V} \mathbb{L}^{N}$ and $\underline{B} \in \mathbb{L}^{N}$ in example 2.1, we have $v^{1}(\{\{1,2\}\})=v(\{1,2\})$.

Also induced LS-games for induced LS-games and so on are possible. We will not go into this in detail. Note that, for all $N \in \mathcal{N}^{\text {dis }}$, each TU-game $(N, v) \in \mathbb{V}^{N}$ corresponds to an LS-game $\left(N, v, \underline{\mathcal{B}_{0}}\right) \in \mathbb{V} \mathbb{L}^{N}$ with a trivial level structure $\mathcal{B}_{0}$ : $=\left\{\mathcal{B}^{0}, \mathcal{B}^{1}\right\}$ and we would like to point out that each LS-game $(N, v, \underline{\mathcal{B}}) \in \mathbb{\mathbb { V }}^{N}$ corresponds to a game with coalition structure (Aumann and Drèze, 1974; Owen, 1977) if $\underline{\mathcal{B}}:=\left\{\mathcal{B}^{0}, \mathcal{B}^{1}, \mathcal{B}^{2}\right\}$.

For all $N \in \mathcal{N}^{\text {dis }}$, an LS-value $\varphi$ is an operator on $\mathbb{V} \mathbb{L}^{N}$ that assigns to any LSgame $(N, v, \underline{\mathcal{B}}) \in \mathbb{V} \mathbb{L}^{N}$ a payoff vector $\varphi(N, v, \underline{\mathcal{B}}) \in \mathbb{R}^{N}$.

By $W^{\text {dis }} \subseteq W$, we define the collection of all positive weight systems on $\mathfrak{U}$ where, for each $w \in W^{\text {dis }}$, we have $w_{i}=w_{j}$ for all $i, j \in \mathfrak{U}$ if $i$ and $j$ have the same submembers. This means that, e.g., for an $\operatorname{LS}$-game $(N, v, \underline{\mathcal{B}}) \in \mathbb{V} \mathbb{L}^{N}, N \in \mathcal{N}^{\text {dis }}$, we have that the weight $w_{i}$ of a player $i \in N$ is equal to the weight $w_{\{i\}}$ of the singleton $\{i\} \in \mathcal{B}^{0}$ in the zeroth LS-game.

We also want a corresponding relationship for sharing systems. The collection $\Lambda^{\text {dis }} \subseteq \Lambda$ of all sharing systems $\lambda \in \Lambda^{\text {dis }}$ on $\mathcal{N}^{\text {dis }}$ is defined by

\footnotetext{
5 In Owen (1977), a corresponding game, for the special case $r=1$ and a level structure $\underline{\mathcal{B}}=\left\{\mathcal{B}^{0}, \mathcal{B}^{1}, \mathcal{B}^{2}\right\}$, is called a quotient game.
} 
$\Lambda^{\mathrm{dis}}:=\left\{\lambda=\left(\lambda_{S, i}\right)_{S \in \mathcal{N}^{\mathrm{dis}}, i \in S} \mid \sum_{i \in S} \lambda_{S, i}=1\right.$ and $\lambda_{S, i} \geq 0$ for each $S \in \mathcal{N}^{\mathrm{dis}}$ and all $i \in S$ such that for all $S^{\prime} \in \mathcal{N}^{\text {dis }}$ with $|S|=\left|S^{\prime}\right|$ and for each $i \in S$ we have a $j \in S^{\prime}$ which has the same sub-members as $i$, we have $\left.\lambda_{S, i}=\lambda_{S^{\prime}, j}\right\}$.

This means that if we have two coalitions with the same number of players, and for every player in the first coalition we have a player in the second coalition with the same sub-members, then the related players should also have the same weight for their respective coalitions.

\subsection{Axioms}

We refer to the following axioms for LS-values $\varphi$ which are mostly simple adaptations of standard axioms for TU-values. All axioms below apply for all $N \in \mathcal{N}^{\text {dis }}$. We do not repeat this phrase at the beginning of each axiom.

Efficiency, $\boldsymbol{E}$. For all $(N, v, \underline{\mathcal{B}}) \in \mathbb{V} \mathbb{L}^{N}$, we have $\sum_{i \in N} \varphi_{i}(N, v, \underline{\mathcal{B}})=v(N)$.

Efficiency requires that the sum of the payoffs should result in the total worth of the game. The next property means that a player who contributes nothing to any coalition should obtain no payoff.

Null player, $N$. For all $(N, v, \underline{B}) \in \mathbb{V} \mathbb{L}^{N}$ and $i \in N$ such that $i$ is a null player in $(N, v)$, we have $\varphi_{i}(N, v, \underline{\mathcal{B}})=0$.

Additivity, $\quad \boldsymbol{A}$. For all $(N, v, \underline{\mathcal{B}}),\left(N, v^{\prime}, \underline{\mathcal{B}}\right) \in \mathbb{V}^{N}$, we have $\varphi(N, v, \underline{\mathcal{B}})+\varphi\left(N, v^{\prime}, \underline{\mathcal{B}}\right)=\varphi\left(N, v+v^{\prime}, \underline{\mathcal{B}}\right)$.

Additivity states that a player's payoff from the sum of two games is the sum of the player's payoff for the two games. By the following axiom, all players receive a non-negative payoff if all coalitions have non-negative dividends.

Positivity, Pos (Vasil'ev, 1975). For all $(N, v, \underline{\mathcal{B}}) \in \mathbb{V} \mathbb{L}^{N}$ such that $(N, v)$ is totally positive, we have $\varphi_{i}(N, v, \underline{\mathcal{B}}) \geq 0$ for all $i \in N$.

Strict monotonicity, SMon (Megiddo, 1974). For all $(N, v, \underline{\mathcal{B}}) \in \mathbb{V} \mathbb{L}^{N}$ and $\alpha \in$ $\mathbb{R}_{++}$, we have $\varphi_{i}\left(N, v+\alpha \cdot u_{N}, \underline{\mathcal{B}}\right)>\varphi_{i}(N, v, \underline{\mathcal{B}})$ for all $i \in N$.

This property means that if the grand coalition improves its worth and the worth of all other coalitions remains the same, all players should receive a higher payoff. In our investigations, the next axiom plays an important role. It claims that the sum of all players' payoffs of a component coincides with this component's payoff in an induced LS-game where the component is regarded as a player.

Level game property, LG (Winter, 1989). For all $(N, v, \underline{\mathcal{B}}) \in \mathbb{V} \mathbb{L}^{N}, \underline{\mathcal{B}}=$ $\left\{\mathcal{B}^{0}, \ldots, \mathcal{B}^{h+1}\right\}, B \in \mathcal{B}^{r}, 0 \leq r \leq h$, we have

$$
\sum_{i \in B} \varphi_{i}(N, v, \underline{\mathcal{B}})=\varphi_{B}\left(\mathcal{B}^{r}, v^{r}, \underline{\mathcal{B}^{r}}\right)
$$

The following axiom says that if two siblings are symmetric in the induced $r$ th LSgame, the total payoff to all players of the first sibling is equal to the total payoff to all players of the second sibling. 
Symmetry between components, Sym $^{6}$ Winter (1989). For all $(N, v, \underline{\mathcal{B}}) \in$ $\mathbb{V} \mathbb{L}^{N}, \underline{\mathcal{B}}=\left\{\mathcal{B}^{0}, \ldots, \mathcal{B}^{h+1}\right\}$, two siblings $B_{k}, B_{\ell} \in \mathcal{B}^{r}, 0 \leq r \leq h$, such that $B_{k}, B_{\ell}$ are symmetric in $\left(\mathcal{B}^{r}, v^{r}\right) \in \mathbb{V}^{\mathcal{B}^{r}}$, we have

$$
\sum_{i \in B_{k}} \varphi_{i}(N, v, \underline{\mathcal{B}})=\sum_{i \in B_{\ell}} \varphi_{i}(N, v, \underline{\mathcal{B}}) .
$$

\subsection{The Shapley levels value}

The following formula is presented in Calvo et al. (1996, Eq. (1)).

For all $N \in \mathcal{N}^{\text {dis }},(N, v, \underline{\mathcal{B}}) \in \mathbb{V}^{N}, \underline{\mathcal{B}}=\left\{\mathcal{B}^{0}, \ldots, \mathcal{B}^{h+1}\right\}$, and $T \in \Omega^{N}, T \ni i$, define

$$
K_{\underline{\mathcal{B}}, T}(i):=\prod_{r=0}^{h} K_{\underline{\mathcal{B}}, T}^{r}(i), \quad \text { where } K_{\underline{\mathcal{B}}, T}^{r}(i):=\frac{1}{\left|\left\{B \in \mathcal{B}^{r}: B \subseteq \mathcal{B}^{r+1}(i), B \cap T \neq \emptyset\right\}\right|}
$$

be the reciprocal cardinality of the set that contains all children of the component of the $(r+1)$ th level containing player $i$, which contain at least one player from coalition $T$. Then, the Shapley levels value ${ }^{7}$ (Winter, 1989) $S h^{L}$ assigns to any $(N, v, \underline{B})$ a vector in $\mathbb{R}^{N}$ defined as

$$
\operatorname{Sh}_{i}^{L}(N, v, \underline{\mathcal{B}}):=\sum_{T \subseteq N, T \ni i} K_{\underline{\mathcal{B}}, T}(i) \Delta_{v}(T) \text { for all } i \in N .
$$

It is easy to see that $S h^{L}$ coincides with $S h$ if $\underline{\mathcal{B}}=\mathcal{B}_{0}$. Winter (1989) used the Owen value (Owen, 1977) as a starting point for his $\overline{\mathrm{LS}}$-value. Therefore, Winter has extended the efficiency, null player, symmetry and additivity axioms to axioms for LS-values. He splits symmetry into symmetry between components and an individual symmetry axiom. If we define a level structure as above, i. e., the singletons are the elements of the lowest level, Winter (1989, remark 1.6) pointed out that we can omit the individual symmetry axiom. In this sense, we present Winter's first axiomatization of the Shapley levels value ${ }^{8}$.

Theorem 2.2 (Winter, 1989) $S h^{L}$ is the unique LS-value that satisfies $\boldsymbol{E}, \boldsymbol{N}, \mathbf{S y m}$, and $\boldsymbol{A}$.

It should be noted that there exist some further axiomatizations of the Shapley levels value by Calvo et al. (1996), Khmelnitskaya and Yanovskaya (2007), Casajus (2010), Álvarez-Mozos and Tejada (2011), and Besner (2019).

\footnotetext{
6 This axiom is called coalitional symmetry in Winter (1989).

7 The value is also known as level(s) structure value or Winter's (Shapley type) value. Our designation is used, e. g., in Álvarez-Mozos et al. (2017)

${ }^{8}$ Winter (1989) introduced his value axiomatically and used this axiomatization as a definition.
} 


\section{Harsanyi support levels solutions}

For TU-games, there are sometimes compelling reasons, not included in the coalition function itself, to treat symmetric players differently. We can have fixed, but for each coalition possibly different, metrics for each player, representing, e. g., the effort, commitment, qualification, or power of a player. Sometimes a player's influence on other players is stronger when another player is not on the team. Or a player can only exert its influence if specific other players have joined the coalition to support this player. The same reasoning can be applied to LS-games, where at different levels the components can be considered as players. With the Shapley levels value, the members of symmetric siblings always get the same payoff in total (Sym). The following LS-values provide the opportunity to take into account the previously mentioned external influences that are not reflected in the coalition function.

Before we give a formal definition, we describe the payoff algorithm: Let $N \in$ $\mathcal{N}^{\text {dis }},(N, v, \underline{\mathcal{B}}) \in \mathbb{V} \mathbb{L}^{N}, \underline{\mathcal{B}}=\left\{\mathcal{B}^{0}, \ldots, \mathcal{B}^{h+1}\right\}$, and $\lambda \in \Lambda^{\text {dis }}$. By (2), $v$ can be expressed as a linear combination of unanimity games. Thus, we have a game $v_{T}:=\Delta_{v}(T) u_{T}$ for each coalition $T \in \Omega^{N}$ which means that we have $\Delta_{v_{T}}(T)=\Delta_{v}(T), \Delta_{v_{T}}(S)=0$ for all $S \subseteq N, S \neq T$, and $v=\sum_{T \in \Omega^{N}} v_{T}$.

In the game $v_{T}$, each component $B^{h}$ of the $h$ th level that contains at least one player $i \in T$ receives a share of $\Delta_{v}(T)$, proportional to its sharing weight $\lambda_{\mathcal{C}_{T}^{h}, B^{h}}$, where $\mathcal{C}_{T}^{h}$ is the coalition that contains all such components $B^{h}$ as elements. Then the share of each component $B^{h}$ involved is distributed among all its children $B^{h-1} \subseteq B^{h}$ of the $(h-1)$ th level, containing at least one player $i \in T$, proportional to their sharing weights $\lambda_{\mathcal{C}_{T}^{h-1}, B^{h-1}}$, where $\mathcal{C}_{T}^{h-1}$ is the coalition that contains all such siblings $B_{h-1}$ as elements, and so on for all levels. In the end, each player $i \in T$ gets its share, "supported" by the sharing weights of all its ancestors. To receive the total payoff of a player, these payoffs are finally added up over all games $v_{T}$ where the player is not a null player.

Definition 3.1 For all $N \in \mathcal{N}^{\text {dis }},(N, v, \underline{\mathcal{B}}) \in \mathbb{V} \mathbb{L}^{N}, \underline{\mathcal{B}}=\left\{\mathcal{B}^{0}, \ldots, \mathcal{B}^{h+1}\right\}, \lambda \in \Lambda^{\mathrm{dis}}$, and $T \in \Omega^{N}, T \ni i, 0 \leq r \leq h$, let

$$
\mathcal{C}_{T}^{r}(i):=\left\{B: B \in \mathcal{B}^{r}, B \subseteq \mathcal{B}^{r+1}(i), B \cap T \neq \emptyset\right\}
$$

be the set that contains all children of the component of the $(r+1)$ th level containing player $i$, which contain at least one player from coalition $T$. Define

$$
K_{\underline{\mathcal{B}}, T}^{\lambda}(i):=\prod_{r=0}^{h} \lambda_{\mathcal{C}_{T}^{r}(i), \mathcal{B}^{r}(i)} .
$$

Then, for each $\lambda \in \Lambda^{\text {dis }}$, the Harsanyi support levels solution $H^{\lambda S L}$ assigns to any $(N, v, \underline{\mathcal{B}})$ a vector in $\mathbb{R}^{N}$ defined as 


$$
H_{i}^{\lambda S L}(N, v, \underline{\mathcal{B}})=\sum_{T \subseteq N, T \ni i} K_{\underline{\mathcal{B}}, T}^{\lambda}(i) \Delta_{v}(T) \text { for all } i \in N .
$$

The class of all Harsanyi support levels solutions is called Harsanyi support levels set and is denoted by $\mathcal{H}^{S L}$.

Remark 3.2 We see that the Shapley levels value is a Harsanyi support levels solution with a $\lambda \in \Lambda^{\text {dis }}$ such that we have for each $\lambda_{\mathcal{C}_{T}^{r}(i), \mathcal{B}^{r}(i)}$ in (10) a $K_{\mathcal{B}, T}^{r}(i)$ in (7) with $\lambda_{\mathcal{C}_{T}^{r}(i), \mathcal{B}^{r}(i)}=K_{\underline{\mathcal{B}}, T}^{r}(i)$.

Remark 3.3 For each $N \in \mathcal{N}^{\text {dis }},(N, v, \underline{\mathcal{B}}) \in \mathbb{V}^{N}, \lambda \in \Lambda^{\text {dis }}$, and for all $T \in \Omega^{N}$, $T \ni i$, we have $\sum_{i \in T} K_{\underline{\mathcal{B}}, T}^{\lambda}(i)=1$ and $K_{\underline{\mathcal{B}}, T}^{\lambda}(i) \geq 0$. Therefore, for fixed $N$ and $\underline{\mathcal{B}} \in \mathbb{L}^{N}$, each $H^{\lambda S L} \in \mathcal{H}^{S L}$ on $(N, v, \underline{\mathcal{B}})$ coincides with a $H^{\lambda^{\prime}} \in \mathcal{H}, \lambda^{\prime} \in \Lambda$, on $(N, v) \in \mathbb{V}^{N}$, where $\lambda_{T, i}^{\prime}=K_{\underline{\mathcal{B}}, T}^{\lambda}(i)$ for each $T \in \Omega^{N}$ and all $i \in T$ and the level structure is disregarded.

To prepare our main result, we first assume that a sharing system $\lambda \in \Lambda^{\text {dis }}$ is exogenously given. We use a technical property for unanimity games with some factor.

Let $\lambda \in \Lambda^{\text {dis }}$. Then an LS-value $\varphi$ satisfies $\lambda$-balanced sharing between components in unanimity games (with some factor), $\boldsymbol{S} \boldsymbol{C} \boldsymbol{U}^{\lambda}$, if for each $N \in$ $\mathcal{N}^{\text {dis }},(N, v, \underline{\mathcal{B}}) \in \mathbb{V} \mathbb{L}^{N}, \underline{\mathcal{B}}=\left\{\mathcal{B}^{0}, \ldots, \mathcal{B}^{h+1}\right\}$, and for all $\alpha \in \mathbb{R}$ and $T \in \Omega^{N}$, two siblings $\quad B_{k}, B_{\ell} \in \mathcal{B}^{r}, 0 \leq r \leq h, B_{k}, B_{\ell} \cap T \neq \emptyset$, and $\mathcal{C}_{T}^{r}\left(B_{k}\right):=\left\{B: B \in \mathcal{B}^{r}, B \subseteq \mathcal{B}^{r+1}\left(B_{k}\right), B \cap T \neq \emptyset\right\}$, we have

$$
\lambda_{\mathcal{C}_{T}^{r}\left(B_{k}\right), B_{\ell}} \sum_{i \in B_{k}} \varphi_{i}\left(N, \alpha \cdot u_{T}, \underline{\mathcal{B}}\right)=\lambda_{\mathcal{C}_{T}^{r}\left(B_{k}\right), B_{k}} \sum_{i \in B_{\ell}} \varphi_{i}\left(N, \alpha \cdot u_{T}, \underline{\mathcal{B}}\right) .
$$

This property says that for two siblings which contain both at least one player from $T$ the ratio of the payoff totals to all players of the two siblings in the unanimity game is equal to the ratio of the sharing weights of both siblings. The sharing weights refer to the partition that has as elements all siblings containing players from $T$.

The following proposition lists some axioms that are satisfied by a Harsanyi support levels solution.

Proposition 3.4 Let $\lambda \in \Lambda^{\mathrm{dis}}$. $H^{\lambda S L}$ satisfies $\boldsymbol{E}, \boldsymbol{N}, \boldsymbol{A}, \boldsymbol{P o s}, \boldsymbol{S C U} \boldsymbol{U}^{\lambda}$, and $\boldsymbol{L G}$.

We present a first axiomatization that replaces $\boldsymbol{S y m}$ in Theorem 2.2 by $\boldsymbol{S} \boldsymbol{C} \boldsymbol{U}^{\lambda}$.

Proposition 3.5 Let $\lambda \in \Lambda^{\mathrm{dis}}$. $H^{\lambda S L}$ is the unique LS-value that satisfies $\boldsymbol{E}, \boldsymbol{N}, \boldsymbol{S C} \boldsymbol{U}^{\lambda}$, and $\boldsymbol{A}$.

It follows that the Harsanyi support levels solutions have an exceptional status among extensions of Harsanyi solutions for LS-games. 
Theorem 3.6 An LS-value $\varphi$ coincides for any fixed $N \in \mathcal{N}^{\text {dis }}$ and $\underline{\mathcal{B}} \in \mathbb{Q}^{N}$ with a Harsanyi solution and satisfies $\boldsymbol{L} \boldsymbol{G}$ if and only if $\varphi \in \mathcal{H}^{S L}$.

By Theorem 3.6 and the famous characterization ${ }^{9}$ of the Harsanyi set in Derks et al. (2000) by $\boldsymbol{E}, \boldsymbol{N}, \boldsymbol{P o s}$, and $\boldsymbol{A}$, the following corollary is immediate.

Corollary 3.7 An LS-value $\varphi$ satisfies $\boldsymbol{E}, \boldsymbol{N}, \boldsymbol{P o s}, \boldsymbol{A}$, and $\boldsymbol{L} \boldsymbol{G}$ if and only if $\varphi \in \mathcal{H}^{S L}$.

\section{Weighted Shapley support levels values}

For applications, the class of weighted Shapley values is an important subset of the Harsanyi set. Therefore, we would like to highlight the following subset of the Harsanyi support levels set that extends the class of weighted Shapley values for LS-games. Each possible coalition is assigned a positive weight. In contrary to the weighted Shapley hierarchy levels values (with the exception of the Shapley levels value), these values satisfy the null player property.

Again, we briefly describe how the dividends are distributed by the following LSvalues. Let $N \in \mathcal{N}^{\text {dis }},(N, v, \underline{\mathcal{B}}) \in \mathbb{V}^{N}, \underline{\mathcal{B}}=\left\{\mathcal{B}^{0}, \ldots, \mathcal{B}^{h+1}\right\}$, and $w \in W^{\text {dis }}$. The dividends of each coalition $T \in \Omega^{N}$ are divided as follows: Each component $B^{h}$ of the $h$ th level that contains at least one player $i \in T$ receives a share of $\Delta_{v}(T)$, proportional to its weight $w_{B^{h}}$. Then the share of each component $B^{h}$ involved is distributed among all its children $B^{h-1} \subseteq B^{h}$ containing at least one player $i \in T$, proportional to their weights $w_{B^{h-1}}$, and so on for all levels. In the end, each player $i \in T$ gets its share, "supported" by the weights of all its ancestors.

Definition 4.1 For all $N \in \mathcal{N}^{\text {dis }},(N, v, \underline{\mathcal{B}}) \in \mathbb{V}^{N}, \underline{\mathcal{B}}=\left\{\mathcal{B}^{0}, \ldots, \mathcal{B}^{h+1}\right\}, w \in W^{\text {dis }}$, and for all $T \in \Omega^{N}, T \ni i$, define

$$
K_{\underline{\mathcal{B}}, T}^{w}(i):=\prod_{r=0}^{h} \frac{w_{\mathcal{B}^{r}(i)}}{\sum_{\substack{B \in \mathcal{B}^{r}: B \subseteq \mathcal{B}^{r+1}(i) \\ B \cap T \neq \emptyset}} w_{B}} .
$$

Then, for each $w \in W^{\text {dis }}$, the weighted Shapley support levels value $S h^{w S L}$ assigns to any $(N, v, \underline{\mathcal{B}})$ a vector in $\mathbb{R}^{N}$ defined as

$$
S h_{i}^{w S L}(N, v, \underline{\mathcal{B}})=\sum_{T \subseteq N, T \ni i} K_{\underline{\mathcal{B}}, T}^{w}(i) \Delta_{v}(T) \text { for all } i \in N .
$$

The class of all weighted Shapley support levels values is denoted by $\mathcal{W} \mathcal{S}^{S L}$.

Remark 4.2 If the weights for all coalitions are equal, (13) coincides with (7). Therefore, the Shapley levels value is also a member of the class of the weighted Shapley support levels values.

\footnotetext{
9 A similar axiomatization can be found already in Vasil'ev (1981) (see Vasil'ev and van der Laan, 2002; Dehez, 2017).
} 
Remark 4.3 For all $N \in \mathcal{N}^{\text {dis }}$ and level structures $\underline{\mathcal{B}} \in \mathbb{L}^{N}, \underline{\mathcal{B}}=\left\{\mathcal{B}^{0}, \ldots, \mathcal{B}^{h+1}\right\}$, a weighted Shapley levels value $S h^{w S L}$ coincides with a Harsanyi support levels solution $H^{\lambda S L}$ where we have

$$
\lambda_{\mathcal{C}_{T}^{r}(i), \mathcal{B}^{r}(i)}=\frac{w_{\mathcal{B}^{r}(i)}}{\sum_{\substack{B \in \mathcal{B}^{r}: B \subseteq \mathcal{B}^{r+1}(i) \\ B \cap T \neq \emptyset}}, w_{B}} \text { for all } T \in \Omega^{N} .
$$

$S h^{w S L}$ coincides with $S h^{w}$ if $\underline{\mathcal{B}}=\underline{\mathcal{B}_{0}}$ and, if $\underline{\mathcal{B}}=\left\{\mathcal{B}^{0}, \mathcal{B}^{1}, \mathcal{B}^{2}\right\}$, the $K_{\underline{\mathcal{B}}, T}^{w}(i)$ coincide with the " $\lambda_{i}^{S}$ " given in Dragan (1992, Sec. 2(e)). Therefore, in this case, the $S h^{w S L}$ coincide with the McLean weighted coalition structure values (Levy and Mclean, 1989; McLean, 1991; Dragan, 1992).

Dependent players are always symmetric but not vice versa. Therefore, our next axiom can be seen as a weighted weakening of Sym. The version for TU-values of this axiom comes from Nowak and Radzik (1995, Axiom A4 (w-Mutual Dependence)).

Let $w \in W^{\text {dis }}$. Then an LS-value $\varphi$ satisfies $\mathbf{w}$-weighted dependence between components, $\boldsymbol{D e p}^{w}$, if for all $N \in \mathcal{N}^{\text {dis }},(N, v, \underline{\mathcal{B}}) \in \mathbb{V} \mathbb{L}^{N}, \underline{\mathcal{B}}=\left\{\mathcal{B}^{0}, \ldots, \mathcal{B}^{h+1}\right\}$, two siblings $B_{k}, B_{\ell} \in \mathcal{B}^{r}, 0 \leq r \leq h$, such that $B_{k}, B_{\ell}$ are dependent in $\left(\mathcal{B}^{r}, v^{r}\right) \in \mathbb{V}^{\mathcal{B}^{r}}$, we have

$$
\sum_{i \in B_{k}} \frac{\varphi_{i}(N, v, \underline{\mathcal{B}})}{w_{B_{k}}}=\sum_{i \in B_{\ell}} \frac{\varphi_{i}(N, v, \underline{\mathcal{B}})}{w_{B_{\ell}}}
$$

The meaning of this axiom is that if two siblings are dependent in the $r$ th LS-game the ratio of the sum of all players' payoffs from the two siblings is equal to the ratio of the weights of both siblings. It follows an extension of the mutual dependence axiom in Nowak and Radzik (1995, Axiom 7).

Mutual dependence between components, MDep : For all $N \in \mathcal{N}^{\mathrm{dis}},(N, v, \underline{\mathcal{B}})$, $\left(N, v^{\prime}, \underline{\mathcal{B}}\right) \in \mathbb{V} \mathbb{L}^{N}, \underline{\mathcal{B}}=\left\{\mathcal{B}^{0}, \ldots, \mathcal{B}^{h+1}\right\}$, two siblings $B_{k}, B_{\ell} \in \mathcal{B}^{r}, 0 \leq r \leq h$, such that $B_{k}, B_{\ell}$ are dependent in $\left(\mathcal{B}^{r}, v^{r}\right) \in \mathbb{V}^{\mathcal{B}^{r}}$ and $\left(\mathcal{B}^{r}, v^{\prime r}\right) \in \mathbb{V}^{\mathcal{B}^{r}}$, we have

$$
\left(\sum_{i \in B_{k}} \varphi_{i}(N, v, \underline{\mathcal{B}})\right) \sum_{i \in B_{\ell}} \varphi_{i}\left(N, v^{\prime}, \underline{\mathcal{B}}\right)=\left(\sum_{i \in B_{\ell}} \varphi_{i}(N, v, \underline{\mathcal{B}})\right) \sum_{i \in B_{k}} \varphi_{i}\left(N, v^{\prime}, \underline{\mathcal{B}}\right) \text {. }
$$

This axiom considers games where two siblings are dependent in two induced $r$ th LS-games. Then this axiom states that the ratio of the payoff totals to all players of the siblings remains invariant for such games if none of the sums is zero. The following proposition lists some axioms that are satisfied by a weighted Shapley support levels value.

Proposition 4.4 Let $w \in W^{\mathrm{dis}}$. Sh $h^{w S L}$ satisfies E, N, A, LG, SMon, Dep ${ }^{w}$, and MDep. 
The next proposition can be seen as an extension of characterizations of the weighted Shapley values with exogenously given weights in Nowak and Radzik (1995).

Proposition 4.5 Let $w \in W^{\mathrm{dis}}$. Sh $h^{w S L}$ is the unique LS-value that satisfies $\boldsymbol{E}, \boldsymbol{N}$, $\mathrm{Dep}^{w}$, and $\boldsymbol{A}$.

Our last theorem axiomatizes the class of all weighted Shapley support levels values. It is closely related to an axiomatization of the class of weighted Shapley values in Nowak and Radzik (1995, Theorem 2.4, Remark 2.3).

Theorem 4.6 An LS-value $\varphi$ satisfies $\boldsymbol{E}, \boldsymbol{N}, \boldsymbol{S M o n}, \boldsymbol{A}$, and MDep if and only if $\varphi \in \mathcal{W S}^{S L}$.

\section{Example}

We give a numerical example to compare the distributions for different values. Let $(N, v, \underline{\mathcal{B}}) \in \mathbb{V}^{\mathbb{N}}, N:=\{1,2,3,4,5,6,7,8,9\}$, and $\underline{\mathcal{B}}=\left\{\mathcal{B}^{0}, \mathcal{B}^{1}, \mathcal{B}^{2}, \mathcal{B}^{3}\right\}$, with $\mathcal{B}^{1}$ : $=\{\{1,2\},\{3,4\},\{5,6\},\{7\},\{8,9\}\}, \mathcal{B}^{2}:=\{\{1,2,3,4\},\{5,6,7\},\{8,9\}\} \quad$ (see Fig. 1).

The coalition function $v$ is given by the following dividends:

$$
\begin{array}{lll}
\Delta_{v}(\{3\})=1, & \Delta_{v}(\{1,2\})=2, & \Delta_{v}(\{1,2,3\})=6, \\
\Delta_{v}(\{5,6,7\})=6, & \Delta_{v}(\{1,2,5,7\})=2, & \Delta_{v}(\{1,2,3,8,9\})=5, \\
\Delta_{v}(\{5,6,7,8,9\})=6, & \Delta_{v}(\{1,2,3,5,6,7,8,9\})=12, &
\end{array}
$$

and all other coalitions have zero dividends in $v$.

For reasons of simplicity, we choose a weight system $w \in W^{\text {dis }}$ such that $w_{S}:=$ $|S|$ for all $S \in \Omega^{N}$.

Furthermore, we denote by $i^{\#}$ the number of all sub-members of a player $i \in \mathfrak{U}$. We use a level sharing system $\lambda \in \Lambda^{\text {dis }}$, given by $\lambda_{\mathcal{B}^{2},\{1,2,3,4\}}:=0.4$, $\lambda_{\mathcal{B}^{2},\{5,6,7\}}:=0.3, \lambda_{\mathcal{B}^{2},\{8,9\}}:=0.3$, and, for all other $S \in \mathcal{N}^{\text {dis }}, S \neq S^{\prime}$ such that $\left|S^{\prime}\right|=$

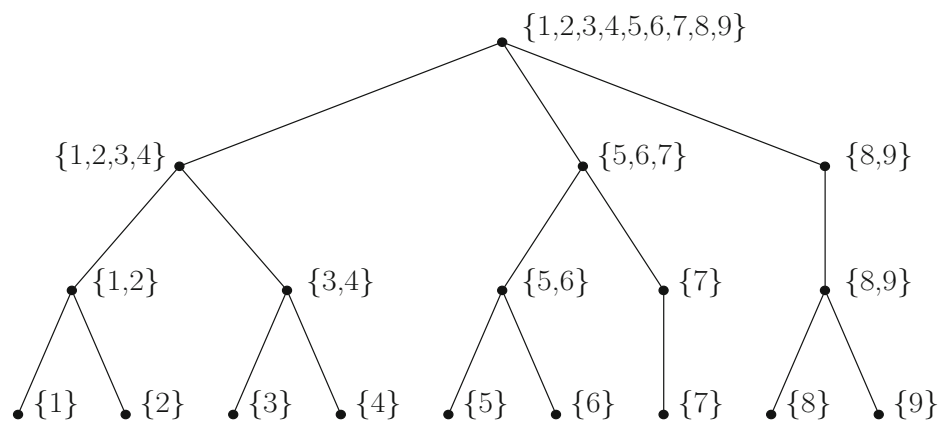

Fig. 1 Structure of the components in different levels 
Table 1 Comparison of different values

\begin{tabular}{llllllllll}
\hline Value & \multicolumn{9}{l}{ Payoff to the players (rounded) } \\
\cline { 2 - 10 } & 1 & 2 & 3 & 4 & 5 & 6 & 7 & 8 & 9 \\
\hline$S h(N, v)$ & 6 & 6 & 5.5 & 0 & 5.2 & 4.7 & 5.2 & 3.7 & 3.7 \\
$S h^{L}(N, v, \underline{\mathcal{B}})$ & 4.625 & 4.625 & 7.25 & 0 & 3.75 & 3.25 & 7 & 4.75 & 4.75 \\
$\operatorname{Sh}^{w H L}(N, v, \underline{\mathcal{B}})$ & 5.1524 & 5.1524 & 7.1690 & 1.3357 & 4.9847 & 4.6524 & 4.8190 & 3.3667 & 3.3667 \\
$\operatorname{Sh}^{w S L}(N, v, \underline{\mathcal{B}})$ & 5.2381 & 5.2381 & 8.3333 & 0 & 5.1048 & 4.5333 & 4.8190 & 3.3667 & 3.3667 \\
$H^{\lambda S L}(N, v, \underline{\mathcal{B}})$ & 5.1048 & 5.1048 & 8.0667 & 0 & 4.9714 & 4.4 & 4.6857 & 3.8333 & 3.8333 \\
\hline
\end{tabular}

$\left|\mathcal{B}^{2}\right|$ and for each $i \in S^{\prime}$ we have a $j \in \mathcal{B}^{2}$ which has the same sub-members as $i$, we have

$$
\lambda_{S, i}:=\frac{i^{\#}}{\sum_{j \in S} j^{\#}} \text { for all } i \in S .
$$

It should be mentioned that here the weighted Shapley hierarchy levels value $S h^{\text {wHL }}$ coincides with the LS-value presented in Gómez-Rúa and Vidal-Puga (2011). We obtain Table 1 . Note that player 4 is a null player, players 1 and 2 and players 8 and 9 respectively are dependent in $(N, v)$, and components $\{5,6\}$ and $\{7\}$ are dependent players in $\left(\mathcal{B}^{1}, v^{1}\right)$.

The payoffs to the dependent players in $(N, v)$ are equal, they have the same weights. The components which are dependent players in $\left(\mathcal{B}^{1}, v^{1}\right)$ have different weights. Thus, the payoffs to players 5 and 6 do not sum up to the payoff to player 7 for $S h^{w H L}, S h^{w S L}$ or $H^{\lambda S L}$. We see that the Shapley levels value benefits player 7 who has not joined a team with other players in the first level. The payoffs to players 8 and 9 are higher by $H^{\lambda S L}$ than by $S h^{w H L}$ or $S h^{w S L}$ since $H^{\lambda S L}$ takes into account the higher weight of the component $\{8,9\}$ within $\mathcal{B}^{2}$ in the level sharing system $\lambda$ compared to that in the weight system $w$.

\section{Conclusion and discussion}

The rapidly increasing volume of collected data and global networking make it possible and necessary to share benefits between cooperating actors, often hierarchically structured. According to the above examinations, for the distribution of the generated surpluses, the presented LS-values of the new classes provide an alternative to the Shapley levels value and the weighted Shapley hierarchy levels values. A close examination of the definition of the Shapley levels value, given by (8), shows that in unanimity games, it is not advantageous for the individual player to merge into components: all components which are siblings have the same weight, even if a sibling is a singleton (see also the payoff to player 7 in Table 1). 
The same need not apply to the LS-values of our new classes and the weighted Shapley hierarchy levels values: the greater the weight of a component, the higher the payoff in a unanimity game. By the weighted Shapley hierarchy levels values, null players also receive a positive payoff if they are part of an involved component (see Table 1) which can be seen as a reward for their contribution to the component's weight.

On the contrary, the LS-values of the Harsanyi support levels set (and, thus, also the weighted Shapley support levels values) always leave null players without benefits. Again, it can be a great competitive advantage for the players to join forces. First, for those who form a cooperating subgroup within the carrier of a unanimity game. And then for all players who belong to coalitions with positive dividends within the whole coalition function. Here, the players of a component can "support" each other in changing unanimity games, even if they do not belong to the carrier. Nevertheless, null players do not receive any payoff, even if they contribute to the total weight of components. However, we can also interpret this in the spirit of cooperative game theory: "As a null player in this game, I support you with all my power, even if I have nothing of it at first. But I know that you as a null player in another game will support me with all your power, even if you have nothing of it there."

This "support" is a fundamental principle of solidarity in all societies. E.g., it forms the business model of all associations and insurance companies. It can be disadvantageous, to kick a null or dummy player out of a group where everyone can rely on each other! This also means that an adaptation for LS-values of the wellknown null player out property in Derks and Haller (1999) is violated, which says that removing a null player from the game does not change the payoff to the other players. An exception is the Shapley levels value. Although it belongs to both the weighted Shapley support levels values and the weighted Shapley hierarchy levels values, null players are not paid and cannot support the corresponding group with their power (weight). Here the groups are powerless in the sense that each group, regardless of size and importance which is not reflected in the coalition function, has the same weight. Despite the fact that additivity is usually considered to be of little economic importance (see, e.g., Casajus and Huettner (2014)), it takes on a new quality in the context of group formation, group solidarity, and the Harsanyi support levels solutions.

\section{Appendix}

\section{Logical independence}

Remark 7.1 The axioms in Corollary 3.7 are logically independent. 


\section{Proof}

- $\boldsymbol{E}, \boldsymbol{N}, \boldsymbol{P o s}, \boldsymbol{A}$ : The axiomatization must also be valid for $\underline{\mathcal{B}}=\underline{\mathcal{B}_{0}}$. In this case, the axioms in Corollary 3.7 coincide with the corresponding axioms for TU-values (and an always satisfied axiom $\boldsymbol{L G}$ ). It is well known that these axioms for TUvalues are logically independent and, therefore, none of the corresponding axioms can be redundant.

- $\boldsymbol{L} \boldsymbol{G}$ : For all $N \in \mathcal{N}^{\text {dis }},(N, v, \underline{\mathcal{B}}) \in \mathbb{V}^{N}, \underline{\mathcal{B}}=\left\{\mathcal{B}^{0}, \ldots, \mathcal{B}^{h+1}\right\}, w \in W^{\text {dis }}$, and for all $T \in \Omega^{N}, T \ni i$, define

$$
A_{\underline{\mathcal{B}}, T}^{w}(i):=\prod_{r=0}^{h} \frac{w_{\mathcal{B}^{r}(i) \cap T}}{\sum_{\substack{B \in \mathcal{B}^{\ell}: B \subseteq \mathcal{B}^{\ell+1}(i), B \cap T \neq \emptyset}} w_{B \cap T}} .
$$

Then, the LS-values $S h^{w A L}$, which assign to any $(N, v, \underline{\mathcal{B}})$ a vector in $\mathbb{R}^{N}$ defined as

$$
S h_{i}^{w A L}(N, v, \underline{\mathcal{B}})=\sum_{T \subseteq N, T \ni i} A_{\underline{\mathcal{B}}, T}^{w}(i) \Delta_{v}(T) \text { for all } i \in N,
$$

satisfy obviously $\boldsymbol{E}, \boldsymbol{N}, \boldsymbol{P o s}$, and $\boldsymbol{A}$, but not $\boldsymbol{L} \boldsymbol{G}$ in general ${ }^{10}$.

Remark 7.2 The axioms in Proposition 4.5 and Theorem 4.6 are logically independent:

Proof The proof is omitted since it is analogous to the proof of Remark 7.1.

\section{Additional axioms and lemmas, used in the proofs}

The first axiom is defined for TU-values, coincides with $\boldsymbol{S C} \boldsymbol{U}^{\lambda}$ in the case of a trivial level structure, and is used in the proof of Theorem 3.6.

$\lambda$-balanced sharing in unanimity games (with some factor), $\boldsymbol{S} \boldsymbol{U}_{T U}^{\lambda}$ : Let $\lambda \in \Lambda$. For all $N \in \mathcal{N},(N, v) \in \mathbb{V}^{N}, \alpha \in \mathbb{R}, T \in \Omega^{N}$, and $i, j \in T$, we have

Table 2 Weights of the coalitions

\begin{tabular}{lcccccccc}
\hline$S$ & $\{1\}$ & $\{2\}$ & $\{3\}$ & $\{4\}$ & $\{5\}$ & $\{1,2\}$ & $\{1,3\}$ & $\{1,4\}$ \\
\hline$w_{S}$ & 1 & 1 & 1 & 1 & 5 & 3 & 3 & 3 \\
\hline$S$ & $\{2,3\}$ & $\{2,4\}$ & $\{3,4\}$ & $\{1,2,3\}$ & $\{1,2,4\}$ & $\{1,3,4\}$ & $\{2,3,4\}$ & $\{1,2,3,4\}$ \\
\hline$w_{S}$ & 3 & 3 & 3 & 5 & 5 & 5 & 5 & 7 \\
\hline
\end{tabular}

${ }^{10}$ Let $\left(N, u_{S}, \underline{\mathcal{B}}\right) \in \mathbb{V} \mathbb{L}^{N}, w \in W^{\text {dis }}$ such that the weights are given as in Table $2, N:=\{1,2,3,4,5\}$, and $\underline{\mathcal{B}}=\left\{\mathcal{B}^{0}, \mathcal{B}^{1}, \mathcal{B}^{2}, \mathcal{B}^{3}\right\}$ with $\mathcal{B}^{1}:=\{\{1,2\},\{3,4\},\{5\}\}, \mathcal{B}^{2}:=\{\{1,2,3,4\},\{5\}\}$, and $\left(N, u_{S}\right)$ be the unanimity game with carrier $S:=\{1,2,3,5\}$. It follows, $\quad S h_{\{1,2,3,4\}}^{w A L}\left(\mathcal{B}^{2}, u_{S}^{2}, \underline{\mathcal{B}^{2}}\right)=\frac{7}{12} \neq \frac{1}{2}=$ $\sum_{i \in\{1,2,3,4\}} S h_{i}^{w A L}\left(N, u_{S}, \underline{\mathcal{B}}\right)$ and $\boldsymbol{L} \boldsymbol{G}$ is not satisfied. 


$$
\lambda_{T, j} \phi_{i}\left(N, \alpha \cdot u_{T}\right)=\lambda_{T, i} \phi_{j}\left(N, \alpha \cdot u_{T}\right) .
$$

The following axiom is a weakening of $\boldsymbol{S C} \boldsymbol{U}^{\lambda}$ and is also used in the proof of Theorem 3.6. Property (12) has to be satisfied only for singletons.

$\lambda$-balanced sharing between singletons in unanimity games (with some factor), $\boldsymbol{S S U} \boldsymbol{U}^{\lambda}$ : Let $\lambda \in \Lambda^{\text {dis }}$. For each $N \in \mathcal{N}^{\text {dis }},(N, v, \underline{\mathcal{B}}) \in \mathbb{V} \mathbb{L}^{N}, \underline{\mathcal{B}}=\left\{\mathcal{B}^{0}, \ldots, \mathcal{B}^{h+1}\right\}$, and for all $\alpha \in \mathbb{R}$ and $T \in \Omega^{N}, i, j \in T$ such that $j \in \mathcal{B}^{1}(i)$, and $\mathcal{C}_{T}^{0}(\{i\}):=\left\{B: B \in \mathcal{B}^{0}, B \subseteq \mathcal{B}^{1}(i), B \cap T \neq \emptyset\right\}$, we have

$$
\lambda_{\mathcal{C}_{T}^{0}(\{i\}),\{j\}} \varphi_{i}\left(N, \alpha \cdot u_{T}, \underline{\mathcal{B}}\right)=\lambda_{\mathcal{C}_{T}^{0}(\{i\}),\{i\}} \varphi_{j}\left(N, \alpha \cdot u_{T}, \underline{\mathcal{B}}\right) .
$$

Our first lemma states that each non-empty coalition $S$ for each level is a subset of only one coalition that is a union of components from this level which have a nonempty intersection with $S$.

Lemma 7.3 Let $N \in \mathcal{N}^{\text {dis }},(N, v, \underline{\mathcal{B}}) \in \mathbb{V} \mathbb{L}^{N}$, and $\underline{\mathcal{B}}=\left\{\mathcal{B}^{0}, \ldots, \mathcal{B}^{h+1}\right\}$. For each level $\mathcal{B}^{r} \in \underline{\mathcal{B}}, 0 \leq r \leq h$, each $S \in \Omega^{N}$ is a subset of exactly one coalition $T^{r} \in \Omega^{N}, T^{r}=$

$$
\begin{aligned}
& \bigcup_{B} \subseteq T^{r}, B \in \mathcal{B}^{r}, B . \text { Thus, we can also uniquely designate each } S \in \Omega^{N} \text { as } S_{T^{r}} \text {. } \\
& \qquad \cap S \neq \emptyset
\end{aligned}
$$

Proof Each coalition $T^{r} \in \Omega^{N}$ is a union of components $B \in \mathcal{B}^{r} . \mathcal{B}^{r}$ is a partition, and so each player $i \in S, S \in \Omega^{N}$, is contained in only one component $B \in \mathcal{B}^{r}$. Therefore, for each coalition $S \in \Omega^{N}$ there is exactly one coalition $T^{r} \in \Omega^{N}$ which is a union of all components $B \in \mathcal{B}^{r}$ containing at least one player $i \in S$.

The second lemma shows that for each coalition $\mathcal{T}$ in an induced level structure the dividend in the induced LS-game is equal to the sum of the dividends in the original game from all coalitions $S$ of the original level structure which are subsets of a coalition $T$ associated to $\mathcal{T}$ and have the property of the previous lemma with respect to coalition $T$.

Lemma 7.4 Let $N \in \mathcal{N}^{\text {dis }},(N, v, \underline{\mathcal{B}}) \in \mathbb{V} \mathbb{L}^{N}, \underline{\mathcal{B}}=\left\{\mathcal{B}^{0}, \ldots, \mathcal{B}^{h+1}\right\}, \mathcal{B}^{r} \in \underline{\mathcal{B}}, 0 \leq r \leq h$, and $S_{T^{r}}$ be the coalitions from lemma 7.3 with associated coalition $T^{r}$. Then, we have in the rth LS-game $\left(\mathcal{B}^{r}, v^{r}, \underline{\mathcal{B}}^{r}\right)$ for each $\mathcal{T}^{r} \in \Omega^{\mathcal{B}^{r}}$, associated to a $T^{r} \in \Omega^{N}$,

$$
\Delta_{v^{r}}\left(\mathcal{T}^{r}\right)=\sum_{S_{T^{r} \subseteq T^{r}}} \Delta_{v}\left(S_{T^{r}}\right)
$$

Proof Let $t=\left|\left\{B \in \mathcal{B}^{r}: B \subseteq T^{r}\right\}\right|$ the number of components $B \in \mathcal{B}^{r}$ which are subsets from a coalition $T^{r} \in \Omega^{N}$ with associated $\mathcal{T}^{r} \in \Omega^{\mathcal{B}^{r}}$. We use induction on the size $t, 1 \leq t \leq\left|\mathcal{B}^{r}\right|$.

Initialization: Let $t=1$. $T^{r}$ is a component $B \in \mathcal{B}^{r}$. We have 


$$
\Delta_{v^{r}}\left(\mathcal{T}^{r}\right) \underset{(1)}{=} v^{r}\left(\mathcal{T}^{r}\right) \underset{(5)}{=} v\left(T^{r}\right)=\sum_{(1)} \sum_{S \subseteq T^{r}} \Delta_{v}(S) \underset{\text { Lem. }}{=} \sum_{S_{T^{r} \subseteq T^{r}}} \Delta_{v}\left(S_{T^{r}}\right)
$$

Induction step: Assume that (15) holds for an arbitrary $\hat{t} \geq 1(I H)$. Let now $\hat{\mathcal{T}}^{r} \in \Omega^{\mathcal{B}^{r}}$ with associated $\hat{T}^{r} \in \Omega^{N}, \hat{t}=\left|\left\{B \in \mathcal{B}^{r}: B \subseteq \hat{T}^{r}\right\}\right|$ and $T^{r}=\hat{T}^{r} \cup \hat{B}, \hat{B} \in \mathcal{B}^{r}, \hat{B} \nsubseteq \hat{T}^{r}$. We have $t=\hat{t}+1$ and it follows

$$
\begin{aligned}
& \Delta_{v^{r}}\left(\mathcal{T}^{r}\right) \quad \underset{(1)}{=} \quad \sum_{\mathcal{Q}^{r} \subseteq \mathcal{T}^{r}} \Delta_{v^{r}}\left(\mathcal{Q}^{r}\right) \underset{(1)}{=} v\left(T^{r}\right)-\sum_{\mathcal{Q}^{r} \subsetneq \mathcal{T}^{r}} \Delta_{v^{r}}\left(\mathcal{Q}^{r}\right) \\
& \underset{(\text { IH })}{(\overline{=})} \quad \Delta_{v}\left(T^{r}\right)+\sum_{S \subsetneq T^{r}} \Delta_{v}(S)-\sum_{\substack{Q^{r} \subsetneq T^{r}, \mathcal{Q}^{r} \subseteq \mathcal{B}^{r}}} \sum_{S_{Q^{r}} \subseteq Q^{r}} \Delta_{v}\left(S_{Q^{r}}\right) \\
& \mathcal{Q}^{r} \subseteq \mathcal{B}^{r} \\
& \underset{\text { Lem. }}{=} \quad \Delta_{v}\left(T^{r}\right)+\sum_{S \subsetneq T^{r}} \Delta_{v}(S)-\sum_{S \subsetneq T^{r},} \Delta_{v}(S) \\
& =\quad \Delta_{v}\left(T^{r}\right)+\sum_{S_{T^{r} \subsetneq T^{r}}} \Delta_{v}\left(S_{T^{r}}\right)=\sum_{S_{T^{r} \subseteq T^{r}}} \Delta_{v}\left(S_{T^{r}}\right) .
\end{aligned}
$$

\section{Proofs}

\section{Proof of Proposition 3.4}

Let $N \in \mathcal{N}^{\text {dis }},(N, v, \underline{\mathcal{B}}) \in \mathbb{V} \mathbb{L}^{N}, \underline{\mathcal{B}}=\left\{\mathcal{B}^{0}, \ldots, \mathcal{B}^{h+1}\right\}, \lambda \in \Lambda^{\text {dis }}$, and $K_{\underline{\mathcal{B}}, T}^{\lambda}$ be the expressions according to Def. 3.1.

- $\boldsymbol{E}, \boldsymbol{N}, \boldsymbol{A}, \boldsymbol{P o s}$ : It is well known that all $H^{\lambda} \in \mathcal{H}, \lambda \in \Lambda$, satisfy the mentioned axioms for TU-values. Thus, the claim follows by Remark 3.3.

- $\boldsymbol{L} \boldsymbol{G}$ : Let $B \in \mathcal{B}^{r}, 0 \leq r \leq h$. If $r=0$, (6) trivially is satisfied because the zeroth LS-game corresponds to the original LS-game.

Let now $1 \leq r \leq h$. Obviously, by (9), we have for all $S \subseteq N, S \cap B \neq \emptyset$,

$$
\sum_{j \in B, j \in S} \prod_{\ell=0}^{r-1} \lambda_{\mathcal{C}_{S}^{\ell}(j), \mathcal{B}^{\ell}(j)}=1
$$

In the game $\left(\mathcal{B}^{r}, v^{r}, \underline{\mathcal{B}^{r}}\right)$, we have for all $\mathcal{T}^{r} \in \Omega^{\mathcal{B}^{r}}, \mathcal{T}^{r} \ni B$, associated $T^{r}$, and an arbitrary $i \in B$,

$$
K_{\underline{\mathcal{B}^{r}, \mathcal{T}^{r}}}^{\lambda}(B) \underset{c(9)}{=} \prod_{\ell=r}^{h} \lambda_{\mathcal{C}_{T^{r}}^{\ell}, \mathcal{B}^{\ell}(i)}
$$

Let $i \in B$ be fixed and $S_{T^{r}} \in \Omega^{N}$ the coalitions from Lemma 7.3 with related 
coalitions $T^{r}$. Note, if $i \in S_{T^{r}}$, we have $B \subseteq T^{r}$. For all $S_{T^{r}}, S_{T^{r}} \ni i, r \leq \ell \leq h$, we have

$$
\lambda_{\mathcal{C}_{S^{r}}^{\ell}(i), \mathcal{B}^{\ell}(i)} \underset{c(9)}{\overline{=}} \quad \lambda_{\mathcal{C}_{T^{r}}^{\ell}(i), \mathcal{B}^{\ell}(i)}
$$

Lem.7.3

It applies, $\mathcal{B}^{\ell}(i)=\mathcal{B}^{\ell}(j)$ for all $j \in B$ and $r \leq \ell \leq h$. For all $S_{T^{r}} \ni i$, it follows,

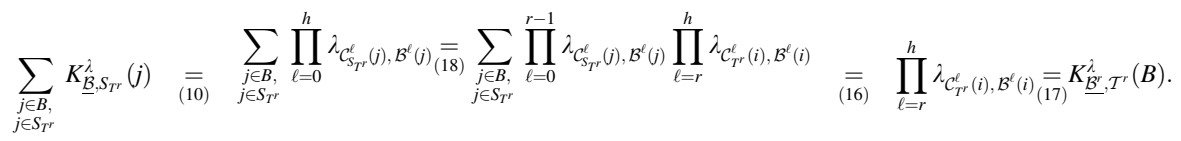

Finally, we get the following:

$$
\begin{aligned}
& \sum_{j \in B} H_{j}^{\lambda S L}(N, v, \underline{\mathcal{B}}) \quad \sum_{(11)}^{=} \quad \sum_{j \in B} K_{\underline{\mathcal{B}}, S}^{\lambda}(j) \Delta_{v}(S) \underset{\substack{L e m \\
7.3}}{=} \sum_{j \in B} \sum_{S_{T^{r}} \subseteq N} K_{\underline{\mathcal{B}}, S_{T^{r}}}^{\lambda}(j) \Delta_{v}\left(S_{T^{r}}\right)
\end{aligned}
$$

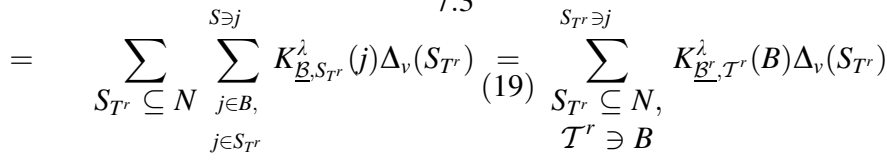

$$
\begin{aligned}
& \begin{array}{l}
= \\
\text { Lem. }
\end{array} \quad \sum_{\mathcal{T}^{r} \subseteq \mathcal{B}^{r}, \mathcal{T}^{r} \ni B} K_{\mathcal{B}^{r}}^{\lambda}, \mathcal{T}^{r}(B) \sum_{S_{T^{r}} \subseteq T^{r}} \Delta_{v}\left(S_{T^{r}}\right) \\
& 7.3 \\
& \underset{\text { Lem. }}{=} \quad \sum_{\mathcal{T}^{r} \subseteq \mathcal{B}^{r}, \mathcal{T}^{r} \ni B} K_{\mathcal{B}^{r}}^{\lambda}, \mathcal{T}^{r}(B) \Delta_{v^{r}}\left(\mathcal{T}^{r}\right) \underset{(11)}{=} H_{B}^{\lambda S L}\left(\mathcal{B}^{r}, v^{r}, \underline{\mathcal{B}^{r}}\right) . \\
& 7.4
\end{aligned}
$$

- $\quad S C \boldsymbol{U}^{\lambda}: \quad$ Let $\quad \alpha \in \mathbb{R}, \quad T \in \Omega^{N}, k, \ell \in N, \mathcal{B}^{r}(\ell) \subseteq \mathcal{B}^{r+1}(k), 0 \leq r \leq h$, $\mathcal{B}^{r}(k), \mathcal{B}^{r}(\ell) \cap T \neq \emptyset$, and $\mathcal{C}_{T}^{r}\left(\mathcal{B}^{r}(k)\right):=\left\{B: B \in \mathcal{B}^{r}, B \subseteq \mathcal{B}^{r+1}\left(\mathcal{B}^{r}(k)\right), B \cap T \neq \emptyset\right\}$. If $\lambda_{\mathcal{C}_{T}^{r}\left(\mathcal{B}^{r}(k)\right), \mathcal{B}^{r}(k)}=0$ or $\lambda_{\mathcal{C}_{T}^{r}\left(\mathcal{B}^{r}(k)\right), \mathcal{B}^{r}(\ell)}=0$, (12) is satisfied by (10) and (11). Otherwise, if $r=0$, we have,

$$
\frac{H_{k}^{\lambda S L}\left(N, \alpha \cdot u_{T}, \underline{\mathcal{B}}\right)}{\lambda_{\mathcal{C}_{T}^{0}(\{k\}),\{k\}}}=\frac{K_{\underline{\mathcal{B}}, T}^{\lambda}(k)}{{ }_{(11)}} \alpha \underset{\mathcal{C}_{T}^{0}(\{k\}),\{k\}}{(\overline{9})} \frac{K_{\underline{\mathcal{B}}, T}^{\lambda}(\ell)}{\lambda_{\mathcal{C}_{T}^{0}(\{k\}),\{\ell\}}} \alpha=\frac{H_{\ell}^{\lambda S L}\left(N, \alpha \cdot u_{T}, \underline{\mathcal{B}}\right)}{\lambda_{\mathcal{C}_{T}^{0}(\{k\}),\{\ell\}}} .
$$

Analogously, in the $r$ th LS-game, $0 \leq r \leq h$, we have

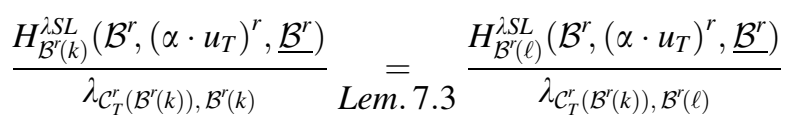

Lem. 7.4

and the claim follows by $\boldsymbol{L} \boldsymbol{G}$. 


\section{Proof of Proposition 3.5}

Let $N \in \mathcal{N}^{\text {dis }},(N, v, \underline{\mathcal{B}}) \in \mathbb{V} \mathbb{L}^{N}, \underline{\mathcal{B}}=\left\{\mathcal{B}^{0}, \ldots, \mathcal{B}^{h+1}\right\}, \lambda \in \Lambda^{\text {dis }}, S \in \Omega^{N}$, and $\varphi$ be an LS-value that satisfies all axioms of Proposition 3.5. Due to Proposition 3.4, (2), and $\boldsymbol{A}$, it is sufficient to show that $\varphi$ is uniquely defined on the game $v_{S}:=\Delta_{v}(S) \cdot u_{S}$. If $B \in \mathcal{B}^{r}, B \cap S=\emptyset$, we have, by $N$,

$$
\sum_{i \in B} \varphi_{i}\left(N, v_{S}, \underline{\mathcal{B}}\right)=0 \text { for all } r, 0 \leq r \leq h .
$$

We use induction on the size $m, 0 \leq m \leq h$, for all levels $r, 0 \leq r \leq h$, with $m:=h-r$.

Initialization: Let $m=0$ and so $r=h$. It follows for all $i \in S$ with $\prod_{\ell=r}^{h} \lambda_{\mathcal{C}_{S}^{\ell}(i), \mathcal{B}^{\ell}(i)}>0$, a such $i$ always exists, and all $B \in \mathcal{B}^{r}, B \cap S \neq \emptyset$, and $B \subseteq \mathcal{B}^{r+1}(i)$,

$$
\begin{gathered}
\sum_{j \in B} \varphi_{j}\left(N, v_{S}, \underline{\mathcal{B}}\right) \underset{S C U^{\lambda}}{=} \frac{\lambda_{\mathcal{C}_{S}^{r}(i), B}^{\lambda_{\mathcal{C}_{S}^{r}(i), \mathcal{B}^{r}(i)}} \sum_{j \in \mathcal{B}^{r}(i)}}{} \varphi_{j}\left(N, v_{S}, \underline{\mathcal{B}}\right) \\
\Rightarrow \sum_{\substack{B \in \mathcal{B}^{r} \\
B \cap S \neq \emptyset}} \sum_{j \in B} \varphi_{j}\left(N, v_{S}, \underline{\mathcal{B}}\right)=\sum_{\substack{B \in \mathcal{B}^{r} \\
B \cap S \neq \emptyset}} \frac{\lambda_{\mathcal{C}_{S}^{r}(i), B}}{\lambda_{\mathcal{C}_{S}^{r}(i), \mathcal{B}^{r}(i)}} \sum_{j \in \mathcal{B}^{r}(i)} \varphi_{j}\left(N, v_{S}, \underline{\mathcal{B}}{\underset{c}{c(20)}}_{E}^{=} \Delta_{v}(S)\right. \\
\Rightarrow \sum_{j \in \mathcal{B}^{r}(i)} \varphi_{j}\left(N, v_{S}, \underline{\mathcal{B}}\right)=\prod_{\ell=r}^{h} \lambda_{\mathcal{C}_{S}^{\ell}(i), \mathcal{B}^{\ell}(i)} \Delta_{v}(S) .
\end{gathered}
$$

By $\boldsymbol{S C} \boldsymbol{U}^{\lambda}$ and (21), we have for all $B \in \mathcal{B}^{r}, B \cap S \neq \emptyset$, and $B \subseteq \mathcal{B}^{r+1}(i)$ with $\prod_{\ell=r}^{h} \lambda_{\mathcal{C}_{S}^{\ell}(i), \mathcal{B}^{\ell}(B)}=0$

$$
\sum_{j \in B} \varphi_{j}\left(N, v_{S}, \underline{\mathcal{B}}\right)=0=\prod_{\ell=r}^{h} \lambda_{\mathcal{C}_{S}^{\ell}(i), \mathcal{B}^{\ell}(B)} \Delta_{v}(S) .
$$

Induction step: Assume that (21) and (22) hold to $\varphi$ with an arbitrary $m-1$, $0 \leq m-1 \leq h-1(I H)$. It follows for all $i \in S$ with $\prod_{\ell=r}^{h} \lambda_{\mathcal{C}_{S}^{\ell}(i), \mathcal{B}^{\ell}(i)}>0$,

$$
\begin{aligned}
& \sum_{\substack{B \in \mathcal{B}^{r}, B \cap S \neq \emptyset \\
B \subseteq \mathcal{B}^{r+1}(i)}} \sum_{j \in B} \varphi_{j}\left(N, v_{S}, \underline{\mathcal{B}}\right){\substack{S C U^{\lambda} \\
=}}_{\substack{B \in \mathcal{B}^{r}, B \cap S \neq \emptyset \\
B \subseteq \mathcal{B}^{r+1}(i)}} \frac{\lambda_{\mathcal{C}_{S}^{r}(i), B}}{\lambda_{\mathcal{C}_{S}^{r}(i), \mathcal{B}^{r}(i)}} \sum_{j \in \mathcal{B}^{r}(i)} \varphi_{j}\left(N, v_{S}, \underline{\mathcal{B}}\right) \\
& B \subseteq \mathcal{B}^{r+1}(i) \\
& \prod_{\ell=r+1}^{h} \lambda_{\mathcal{C}_{S}^{\ell}(i), \mathcal{B}^{\ell}(i)} \Delta_{v}(S) \\
& \Rightarrow \quad \sum_{j \in \mathcal{B}^{r}(i)} \varphi_{j}\left(N, v_{S}, \underline{\mathcal{B}}\right) \\
& \prod_{\ell=r}^{h} \lambda_{\mathcal{C}_{S}^{\ell}(i), \mathcal{B}^{\ell}(i)} \Delta_{v}(S) .
\end{aligned}
$$

Analogously, for all $B \in \mathcal{B}^{r}, B \cap S \neq \emptyset$, with $\prod_{\ell=r}^{h} \lambda_{\mathcal{C}_{S}^{\ell}(i), \mathcal{B}^{\ell}(B)}=0$, we have 


$$
\sum_{j \in B} \varphi_{j}\left(N, v_{S}, \underline{\mathcal{B}}\right)=0=\prod_{\ell=r}^{h} \lambda_{\mathcal{C}_{S}^{\ell}(i), \mathcal{B}^{\ell}(B)} \Delta_{v}(S) .
$$

Therefore, $\varphi$ is uniquely defined on $v_{S}$ (take $m=h$ and so $r=0$ ).

\section{Proof of Theorem 3.6}

Let $N \in \mathcal{N}^{\text {dis }}$ and $\underline{\mathcal{B}} \in \mathbb{L}^{N}$ be fixed.

$\Rightarrow$ : By Remark 3.3 and Proposition 3.4, each $\varphi \in \mathcal{H}^{S L}$ on $(N, v, \underline{\mathcal{B}}) \in \mathbb{V} \mathbb{L}^{N}$ coincides with a Harsanyi solution $\phi \in \mathcal{H}$ on $(N, v) \in \mathbb{V}^{N}$ and satisfies $\boldsymbol{L} \boldsymbol{G}$.

$\Leftarrow$ : Obviously, if we use the coinciding axioms for TU-values in Proposition 3.5, we have characterizations of Harsanyi solutions with exogenously given sharing systems $\lambda \in \Lambda$. Note that these axiomatizations hold for all $N \in \mathcal{N}$ and, especially, for games which are defined on the same player sets as used for induced LS-games of the original LS-game on a fixed $N \in \mathcal{N}^{\text {dis }}, \underline{\mathcal{B}} \in \mathbb{L}^{N}$. Thus, each $\phi \in \mathcal{H}$ satisfies the standard axioms $\boldsymbol{E}_{T U}, \boldsymbol{N}_{T U}, \boldsymbol{A}_{T U}$ for TU-values, and $\boldsymbol{S} \boldsymbol{U}_{T U}^{\lambda}$ for some $\lambda \in \Lambda$. Any LS-value $\varphi$ that coincides with a Harsanyi solution on $N \in \mathcal{N}^{\text {dis }}, \underline{\mathcal{B}} \in \mathbb{L}^{N}$, must also coincide with a Harsanyi solution in the induced LS-games. Therefore, any LS-value $\varphi$ that coincides with a Harsanyi solution must satisfy the simply transferred versions for LS-values of these axioms: $\boldsymbol{E}, \boldsymbol{N}, \boldsymbol{A}$, and $\boldsymbol{S} \boldsymbol{S} \boldsymbol{U}^{\lambda^{\prime}}$ for some $\lambda^{\prime} \in \Lambda^{\text {dis }}$ and also in corresponding induced LS-games. Note that $\boldsymbol{S S} \boldsymbol{U}^{\lambda^{\prime}}$ is implied by $\boldsymbol{S} \boldsymbol{U}^{\lambda}$, and $\boldsymbol{S} \boldsymbol{C} \boldsymbol{U}^{\lambda}$ is implied by $\boldsymbol{S} \boldsymbol{U}^{\lambda^{\prime}}$ and $\boldsymbol{L} \boldsymbol{G}$ for some $\lambda, \lambda^{\prime} \in \Lambda^{\text {dis }}$ for any fixed $N \in \mathcal{N}^{\text {dis }}, \underline{\mathcal{B}} \in \mathbb{L}^{N}$. Thus, all the axioms of Proposition 3.5 must be satisfied and we have $\varphi \in \mathcal{H}^{S L}$.

\section{Proof of Corollary 3.7}

The claim follows immediately due to the axiomatization of the Harsanyi set (Derks et al., 2000) by the versions for TU-values of $\boldsymbol{E}, \boldsymbol{N}, \boldsymbol{P o s}$, and $\boldsymbol{A}$ and Theorem 3.6.

\section{Proof of Proposition 4.4}

Let $N \in \mathcal{N}^{\text {dis }},(N, v, \underline{\mathcal{B}}) \in \mathbb{V} \mathbb{L}^{N}, \underline{\mathcal{B}}=\left\{\mathcal{B}^{0}, \ldots, \mathcal{B}^{h+1}\right\}, w \in W^{\text {dis }}$, and $K_{\underline{\mathcal{B}}, T}^{w}$ be the expressions according to Def. 4.1.

- $\boldsymbol{E}, \boldsymbol{N}, \boldsymbol{A}, \boldsymbol{L} \boldsymbol{G}$ : The claim follows directly by Remark 4.3 and Proposition 3.4.

- SMon : The claim follows immediately by (14).

- Dep ${ }^{w}$ : Let $k, \ell \in N, 0 \leq r \leq h, \mathcal{B}^{r}(\ell) \subseteq \mathcal{B}^{r+1}(k)$ and $\mathcal{B}^{r}(k), \mathcal{B}^{r}(\ell)$ be dependent in $\left(\mathcal{B}^{r}, v^{r}\right) \in \mathbb{V}^{\mathcal{B}^{r}}$. If $r=0$, then $k, \ell$ are dependent in $(N, v) \in \mathbb{V}^{N}$ and we get 


$$
\begin{aligned}
& \frac{S h_{k}^{w S L}(N, v, \underline{\mathcal{B}})}{w_{\{k\}}} \quad \underset{(14)}{=} \sum_{T \subseteq N, T \ni k} \frac{K_{\underline{\mathcal{B}}, T}^{w}(k)}{w_{\{k\}}} \Delta_{v}(T) \quad \underset{{ }^{(3)}}{=} \sum_{T \subseteq N,\{k, \ell\} \subseteq T} \frac{K_{\underline{\mathcal{B}}, T}^{w}(k)}{w_{\{k\}}} \Delta_{v}(T) \\
& c=\sum_{c \overline{D e f} \cdot T \subseteq N,\{k, \ell\} \subseteq T} \frac{K_{\underline{\mathcal{B}}, T}^{w}(\ell)}{w_{\{\ell\}}} \Delta_{v}(T) \quad=\frac{\operatorname{Sh}_{\ell}^{w S L}(N, v, \underline{\mathcal{B}})}{w_{\{\ell\}}} .
\end{aligned}
$$

Thus, we have also in the $r$ th LS-game, $0 \leq r \leq h$,

$$
\frac{S h_{\mathcal{B}^{r}(k)}^{w S L}\left(\mathcal{B}^{r}, v^{r}, \underline{\mathcal{B}^{r}}\right)}{w_{\mathcal{B}^{r}(k)}}=\frac{S h_{\mathcal{B}^{r}(\ell)}^{w S L}\left(\mathcal{B}^{r}, v^{r}, \underline{\mathcal{B}^{r}}\right)}{w_{\mathcal{B}^{r}(\ell)}}
$$

and the claim follows by $\boldsymbol{L} \boldsymbol{G}$.

- MDep : The claim follows immediately by $\boldsymbol{D e p}^{w}$.

Convention 7.5 To avoid cumbersome case distinctions in the proof of Proposition 4.5 , if we consider only one single component isolated as a player, we define the component dependent on itself. Then $\boldsymbol{D e p}^{w}$ is trivially satisfied.

\section{Proof of Proposition 4.5}

Let $N \in \mathcal{N}^{\text {dis }},(N, v, \underline{\mathcal{B}}) \in \mathbb{V} \mathbb{L}^{N}, \underline{\mathcal{B}}=\left\{\mathcal{B}^{0}, \ldots, \mathcal{B}^{h+1}\right\}, w \in W^{\text {dis }}, S \in \Omega^{N}$, and $\varphi$ be an LS-value that satisfies all axioms of Theorem 4.5. Due to Proposition 4.4, (2), and $\boldsymbol{A}$, it is sufficient to show that $\varphi$ is uniquely defined on the game $v_{S}:=\Delta_{v}(S) \cdot u_{S}$.

By Lemma 7.3, for each level $r, 0 \leq r \leq h$, exists exactly one coalition $T_{S}^{r}$ with associated $\mathcal{T}_{S}^{r} \subseteq \mathcal{B}^{r}$, which is the smallest coalition of all $R^{r}, R^{r} \supseteq S$, with associated $\mathcal{R}^{r} \subseteq \mathcal{B}^{r}$ and so in each game $\left(\mathcal{B}^{r}, v_{S}^{r}, \underline{\mathcal{B}^{r}}\right) \in \mathbb{V} \mathbb{L}^{\mathcal{B}^{r}}$, we have $\Delta_{v_{S}^{r}}\left(\mathcal{T}_{S}^{r}\right)=\Delta_{v}(S)$ and $\Delta_{v_{S}^{r}}\left(\mathcal{R}^{r}\right)=0$ for $\mathcal{R}^{r} \subseteq \mathcal{B}^{r}, \mathcal{R}^{r} \neq \mathcal{T}_{S}^{r}$. Therefore, by (3), possibly using Conv. 7.5, all components $B \in \mathcal{B}^{r}, B \cap S \neq \emptyset$, are dependent in $\left(\mathcal{B}^{r}, v_{S}^{r}\right)$. If $B \in \mathcal{B}^{r}, B \cap S=\emptyset$, we have, by $N, \sum_{i \in B} \varphi_{i}\left(N, v_{S}, \underline{\mathcal{B}}\right)=0$.

We use induction on the size $m, 0 \leq m \leq h$, for all levels $r, 0 \leq r \leq h$, with $m:=h-r$.

Initialization: Let $m=0$ and so $r=h$. For an arbitrary $i \in S$, we get

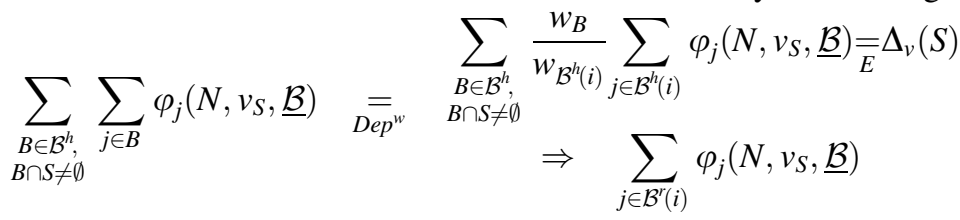

$$
\begin{aligned}
& =\prod_{k=h-m}^{h} \frac{w_{\mathcal{B}^{k}(i)}}{\sum_{\substack{B \in \mathcal{B}^{k}: B \subseteq \mathcal{B}^{k+1}(i), B \cap S \neq \emptyset}} w_{B}} \Delta_{v}(S) .
\end{aligned}
$$

Induction step: Assume that (23) holds to $\varphi$ with an arbitrary $m-1,0 \leq m-$ $1 \leq h-1(I H)$. It follows, for an arbitrary $i \in S$, 


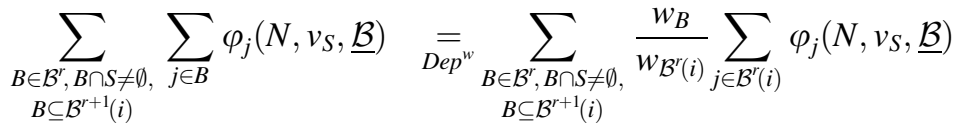

$$
\begin{aligned}
& \underset{(\overline{I H})}{k=h-m+1} \prod_{\substack{B \in \mathcal{B}^{k}: B \subseteq \mathcal{B}^{k+1}(i), B \cap S \neq \emptyset}}^{h} \frac{w_{B}}{\sum_{\mathcal{B}^{k}(i)}} \Delta_{v}(S) \\
& \Rightarrow \quad \sum_{j \in \mathcal{B}^{r}(i)} \varphi_{j}\left(N, v_{S}, \underline{\mathcal{B}}\right) \\
& =\prod_{k=h-m}^{h} \frac{w_{\mathcal{B}^{k}(i)}}{\sum_{\substack{B \in \mathcal{B}^{k}: B \subseteq \mathcal{B}^{k+1}(i), B \cap S \neq \emptyset}} w_{B}} \Delta_{v}(S) .
\end{aligned}
$$

Therefore, $\varphi$ is uniquely defined on $v_{S}$ (take $m=h$ and so $r=0$ ).

\section{Proof of Theorem 4.6}

By Proposition 4.4, we only have to show the way back.

Let $N \in \mathcal{N}^{\text {dis }},(N, v, \underline{\mathcal{B}}) \in \mathbb{V} \mathbb{L}^{N}, \underline{\mathcal{B}}=\left\{\mathcal{B}^{0}, \ldots, \mathcal{B}^{h+1}\right\}$, and $\varphi$ be an LS-value that satisfies $\boldsymbol{E}, \boldsymbol{N}, \boldsymbol{S M o n}, \boldsymbol{A}$, and MDep. By SMon and $\boldsymbol{N}$, we have $\varphi_{i}\left(N, u_{N}, \underline{\mathcal{B}}\right)>0$ for all $i \in N$. Take a $w \in W^{\text {dis }}$ such that $w_{B}:=\sum_{i \in B} \varphi_{i}\left(N, u_{N}, \underline{\mathcal{B}}\right)$ for all $B \in \underline{\mathcal{B}}$. By MDep, we have for all $B_{k}, B_{\ell} \in \mathcal{B}^{r}, 0 \leq r \leq h$, such that $B_{\ell} \subseteq \mathcal{B}^{r+1}\left(B_{k}\right)$, and $B_{k}, B_{\ell}$ are dependent in $\left(\mathcal{B}^{r}, v^{r}\right) \in \mathbb{V}^{\mathcal{B}^{r}}$,

$$
\sum_{i \in B_{k}} \frac{\varphi_{i}(N, v, \underline{\mathcal{B}})}{w_{B_{k}}}=\sum_{i \in B_{\ell}} \frac{\varphi_{i}(N, v, \underline{\mathcal{B}})}{w_{B_{\ell}}}
$$

and $\boldsymbol{D e} \boldsymbol{p}^{w}$ is satisfied. The claim follows by Proposition 4.5.

Acknowledgements We are grateful to two anonymous referees for their helpful comments and suggestions.

Open Access This article is licensed under a Creative Commons Attribution 4.0 International License, which permits use, sharing, adaptation, distribution and reproduction in any medium or format, as long as you give appropriate credit to the original author(s) and the source, provide a link to the Creative Commons licence, and indicate if changes were made. The images or other third party material in this article are included in the article's Creative Commons licence, unless indicated otherwise in a credit line to the material. If material is not included in the article's Creative Commons licence and your intended use is not permitted by statutory regulation or exceeds the permitted use, you will need to obtain permission directly from the copyright holder. To view a copy of this licence, visit http:// creativecommons.org/licenses/by/4.0/.

Funding Open Access funding enabled and organized by Projekt DEAL. 


\section{References}

Álvarez-Mozos, M., van den Brink, R., van der Laan, G., \& Tejada, O. (2017). From hierarchies to levels: new solutions for games with hierarchical structure. International Journal of Game Theory, 1-25.

Álvarez-Mozos, M., \& Tejada, O. (2011). Parallel characterizations of a generalized Shapley value and a generalized Banzhaf value for cooperative games with level structure of cooperation. Decision Support Systems, 52(1), 21-27.

Aumann, R. J., \& Drèze, J. (1974). Cooperative games with coalition structures. International Journal of Game Theory, 3, 217-237.

Besner, M. (2019). Weighted Shapley hierarchy levels values. Operations Research Letters, 47, 122-126.

Calvo, E., \& Gutiérrez, E. (2013). The Shapley-solidarity value for games with a coalition structure. International Game Theory Review, 15(01), 1350002.

Calvo, E., Lasaga, J. J., \& Winter, E. (1996). The principle of balanced contributions and hierarchies of cooperation. Mathematical Social Sciences, 31(3), 171-182.

Casajus, A. (2010). Another characterization of the Owen value without the additivity axiom. Theory and Decision, 69(4), 523-536.

Casajus, A., \& Huettner, F. (2014). Weakly monotonic solutions for cooperative games. Journal of Economic Theory, 154, 162-172.

Dehez, P. (2017). On Harsanyi dividends and asymmetric values. International Game Theory Review, 19(03), 1750012.

del Pozo, M., Manuel, C., González-Arangüena, E., \& Owen, G. (2011). Centrality in directed social networks. A game theoretic approach. Social Networks, 33(3), 191-200.

Derks, J., Haller, H., \& Peters, H. (2000). The selectope for cooperative games. International Journal of Game Theory, 29(1), 23-38.

Derks, J. J., \& Haller, H. H. (1999). Null players out? Linear values for games with variable supports. International Game Theory Review, 1(3-4), 301-314.

Dragan, I. C. (1992). Multiweighted Shapley values and random order values. University of Texas at Arlington.

Driessen, T. S. H., \& Funaki, Y. (1991). Coincidence of and collinearity between game theoretic solutions. Operations Research Spektrum, 13(1), 15-30.

Gómez-Rúa, M., \& Vidal-Puga, J. (2011). Balanced per capita contributions and level structure of cooperation. Top, 19(1), 167-176.

Hammer, P. L., Peled, U. N., \& Sorensen, S. (1977). Pseudo-Boolean functions and game theory. I. Core elements and Shapley value. Cahiers du CERO, 19, 159-176.

Harsanyi, J. C. (1959). A bargaining model for cooperative n-person games. In A. W. Tucker \& R. D. Luce (Eds.), Contributions to the theory of games IV (pp. 325-355). Princeton NJ: Princeton University Press.

Hart, S., \& Mas-Colell, A. (1989). Potential, value, and consistency. Econometrica: Journal of the Econometric Society, 589-614.

Joosten, R. (1996). Dynamics, equilibria and values. The Netherlands: Maastricht University (PhD thesis).

Kalai, E., \& Samet, D. (1987). On weighted Shapley values. International Journal of Game Theory, 16(3), 205-222.

Khmelnitskaya, A. B., \& Yanovskaya, E. B. (2007). Owen coalitional value without additivity axiom. Mathematical Methods of Operations Research, 66(2), 255-261.

Levy, A., \& Mclean, R. P. (1989). Weighted coalition structure values. Games and Economic Behavior, 1(3), 234-249.

McLean, R. P. (1991). Random order coalition structure values. International Journal of Game Theory, 20(2), 109-127.

Megiddo, N. (1974). On the nonmonotonicity of the bargaining set, the kernel and the nucleolus of game. SIAM Journal on Applied Mathematics, 27(2), 355-358.

Nowak, A. S., \& Radzik, T. (1994). A solidarity value for n-person transferable utility games. International Journal of Game Theory, 23(1), 43-48.

Nowak, A. S., \& Radzik, T. (1995). On axiomatizations of the weighted Shapley values. Games and Economic Behavior, 8(2), 389-405.

Owen, G. (1977). Values of games with a priori unions. Essays in mathematical economics and game theory (pp. 76-88). Berlin: Springer. 
Shapley, L. S. (1953a). Additive and non-additive set functions. Princeton University.

Shapley, L. S. (1953b). A value for n-person games. In H. W. Kuhn \& A. W. Tucker (Eds.), Contributions to the theory of games (Vol. 2, pp. 307-317). Princeton: Princeton University Press.

Vasil'ev, V., \& van der Laan, G. (2002). The Harsanyi set for cooperative TU-games. Siberian Advances in Mathematics, 12, 97-125.

Vasil'ev, V. A. (1975). The Shapley value for cooperative games of bounded polynomial variation. Optimizacija Vyp, 17, 5-27.

Vasil'ev, V. A. (1978). Support function of the core of a convex game. Optimizacija Vyp, 21, 30-35.

Vasil'ev, V. A. (1981). On a class of imputations in cooperative games. Soviet Mathematics Dokladi, 23, 53-57.

Vidal-Puga, J. (2012). The Harsanyi paradox and the "right to talk" in bargaining among coalitions. Mathematical Social Sciences, 64(3), 214-224.

Weber, R. J. (1988). Probabilistic values for games. In A. E. Roth (Ed.), The Shapley value, essays in honor of L.S. Shapley (pp. 101-119). Cambridge: Cambridge University Press.

Winter, E. (1989). A value for cooperative games with levels structure of cooperation. International Journal of Game Theory, 18(2), 227-240.

Publisher's Note Springer Nature remains neutral with regard to jurisdictional claims in published maps and institutional affiliations. 